\title{
The effects of magnetic topology on the SOL turbulence transport in the first divertor plasma operation of W7-X using a new combined probe
}

\author{
S. C. Liu ${ }^{1,2}$, Y. Liang ${ }^{1,2}$, P. Drews' ${ }^{2}$, C. Killer ${ }^{3}$, A. Knieps ${ }^{2}$, G. S. Xu ${ }^{1}$, H. Q. Wang ${ }^{1}$, N. Yan ${ }^{1}$, X. Han ${ }^{1,2}$, D. Höschen ${ }^{2}$, \\ A. Krämer-Flecken ${ }^{2}$, D. Nicolai ${ }^{2}$, G. Satheeswaran ${ }^{2}$, K. Hammond ${ }^{3}$, J. Q. Cai ${ }^{1,2}$, A. Charl ${ }^{2}$, J. Cosfeld ${ }^{2}$, G. Fuchert ${ }^{3}$, \\ Y. Gao ${ }^{2}$, J. Geiger ${ }^{3}$, O. Grulke , M. Henkel ${ }^{2}$, M. Hirsch ${ }^{3}$, U. Hoefel ${ }^{3}$, R. König ${ }^{3}$, Y. Li ${ }^{1,2}$, O. Neubauer ${ }^{2}$, E. Pasch ${ }^{3}$, \\ K. Rahbarnia ${ }^{3}$, M. Rack ${ }^{2}$, N. Sandri ${ }^{2}$, S. Sereda ${ }^{2}$, B. Schweer ${ }^{2}$, E. H. Wang ${ }^{1,2}$, S. Xu ${ }^{1,2}$, X. Gao ${ }^{1}$ and W7-X Team \\ ${ }^{1}$ Institute of Plasma Physics, Chinese Academy of Sciences, Hefei 230031, People's Republic of China \\ ${ }^{2}$ Forschungszentrum Jülich GmbH, Institut für Energie- und Klimaforschung - Plasmaphysik, Partner of the Trilateral Euregio \\ Cluster (TEC), 52425 Jülich, Germany \\ ${ }^{3}$ Max-Planck-Institute for Plasma Physics, 17491 Greifswald, Germany
}

Email: 1shch@ipp.ac.cn

\begin{abstract}
Wendelstein 7-X (W7-X) was operated successfully with the first divertor plasma in the operation phase 1.2a (OP1.2a). A new combined probe head, developed and installed on the multiple-purpose manipulator, is able to measure the edge plasma profiles $\left(T_{e}, n_{e}, \phi_{f}, M_{\|}\right)$, variation of magnetic field, poloidal and radial turbulence structures. The scrape-off layer (SOL) plasma parameters in two magnetic configurations (standard and high mirror) are in good agreement with the magnetic island structure and the field line connection length calculated by the field line tracer. In both the standard and high mirror configurations, the radial turbulent heat flux and particle flux have strong dependence on the local magnetic topology, revealing two distinct transport patterns: a broadband turbulence dominant region in the outer SOL and a low frequency dominant region in the inner SOL. In the standard divertor configuration, the broadband turbulence with a frequency range of $40-120 \mathrm{kHz}$ is located near the island center along the probe path, leading to outward transport. These broadband fluctuations propagate with a velocity of $2.3-4.4 \mathrm{~km} / \mathrm{s}$ poloidally along the ion diamagnetic drift direction in the plasma frame, with $k_{\theta} \rho_{s}$ close to 0.1 . The large radial transport induced by the broadband turbulence is accompanied by a steep electron density gradient. The low frequency (5-30 $\mathrm{kHz}$ ) dominant transport exhibits obvious intermittent structure. Some statistical techniques are applied to the characterization of the intermittent transport.
\end{abstract}

\section{Introduction}

The edge cross-field transport in fusion devices is considered to be driven by turbulence, such as anomalous transport which is much larger than neoclassic transport [1]. Usually drift wave turbulence is proposed to drive the anomalous transport, which has been investigated in both theory and experiment [24]. The turbulence can be classified by the dimensionless parameter $k_{\perp} \rho_{S}$, where $k_{\perp}$ is the perpendicular wavenumber and $\rho_{s}$ is the ion sound Larmor radius. The ion temperature gradient (ITG) turbulence is expected to have $k_{\perp} \rho_{s} \approx 0.1-$ 0.5 , and to propagate with phase velocity close to the ion diamagnetic drift velocity in the plasma frame. The ITG instability can be excited when the inequality $\eta_{i} \equiv \partial_{r} \ln T_{i} /$ $\partial_{r} \ln n_{i}>\eta_{\text {crit }}$ is satisfied, with $\eta_{\text {crit }} \sim 1$. The collisionless trapped electron mode (TEM) is driven by the electron pressure gradient, with $k_{\perp} \rho_{S} \sim 1$ and propagating in the electron diamagnetic drift direction in the plasma frame. When the wavenumber of TEM increases to $k_{\perp} \rho_{s}>1$, the TEM transits into the electron temperature gradient (ETG) instability. The ETG can be triggered when $\eta_{e} \equiv \partial_{r} \ln \left(T_{e}\right) /$ $\partial_{r} \ln \left(n_{e}\right)>\eta_{\text {crit }} \sim 1$, and is expected to induce fine-grained turbulence with $k_{\perp} \rho_{s} \sim 1-10$ and propagate along the electron diamagnetic drift direction in the plasma frame [3]. In the plasma edge and the scrape-off layer (SOL), intermittent events are also considered to play an important role in the cross-field transport due to its large scale and large fluctuation amplitude. The intermittent transport, also named as blobs, filaments or eddies, has been measured by several diagnostics in many fusion devices, such as the gas puff imaging (GPI) in NSTX, Alcator C-Mod, TEXTOR and EAST [5-9], beam emission spectroscopy (BES) in DIII-D and KSTAR [10, 11], and Langmuir probes in W7-AS, LHD, TJ-K, JET, ASDEX Upgrade, HL-2A and DIII-D [10, 12-18]. As measured by different machines, the blobs typically have a size of $1-3 \mathrm{~cm}$, 
radial velocity about $0.5-2 \mathrm{~km} / \mathrm{s}$. In TJ-K stellarator, the blobs are demonstrated that they occur in the SOL region with negative mean normal curvature and reveal field-aligned structures, which leads to a significant fraction of the turbulent SOL transport [17]. The blob generation and its radial propagation has been interpreted by the interchange model [19]. In stellarators, the edge transport is largely determined by the 3D magnetic topology. In LHD, the stochastization of magnetic topology not only changes the plasma profiles but also causes the toroidal flow damping and influences the transport $[20,21]$. During a magnetic well scan experiment in TJ-II, the high frequency Alfvénic mode, middle frequency and low frequency oscillations $(10-20 \mathrm{kHz})$ depend on the magnetic topology intensively, and the low frequency oscillation may be induced by the magnetic island rotation [22]. In W7-AS, the electron density fluctuations and plasma confinement are extremely sensitive to the edge rotational transform $\iota_{a}$ which can be modified by either external coils or plasma current [23].

In Wendelstein 7-X (W7-X), the turbulence characteristics also exhibit a strong dependence on magnetic topologies, as measured by a combined probe head in operation phase 1.1 $[24,25]$. During the first island divertor experiment in operation phase 1.2a (OP1.2a), the edge magnetic island structure and radial transport properties have been obtained by a new combined probe designed to measure edge profiles and turbulence structures. The rest of paper is organized as follows. The experimental setup is described in section 2 . The turbulence structures and their magnetic topologies are shown in section 3. The radial transport induced by turbulence in standard divertor configuration is presented in section 4 . Turbulent transport in high mirror configuration is given in section 5. Section 6 is the summary.
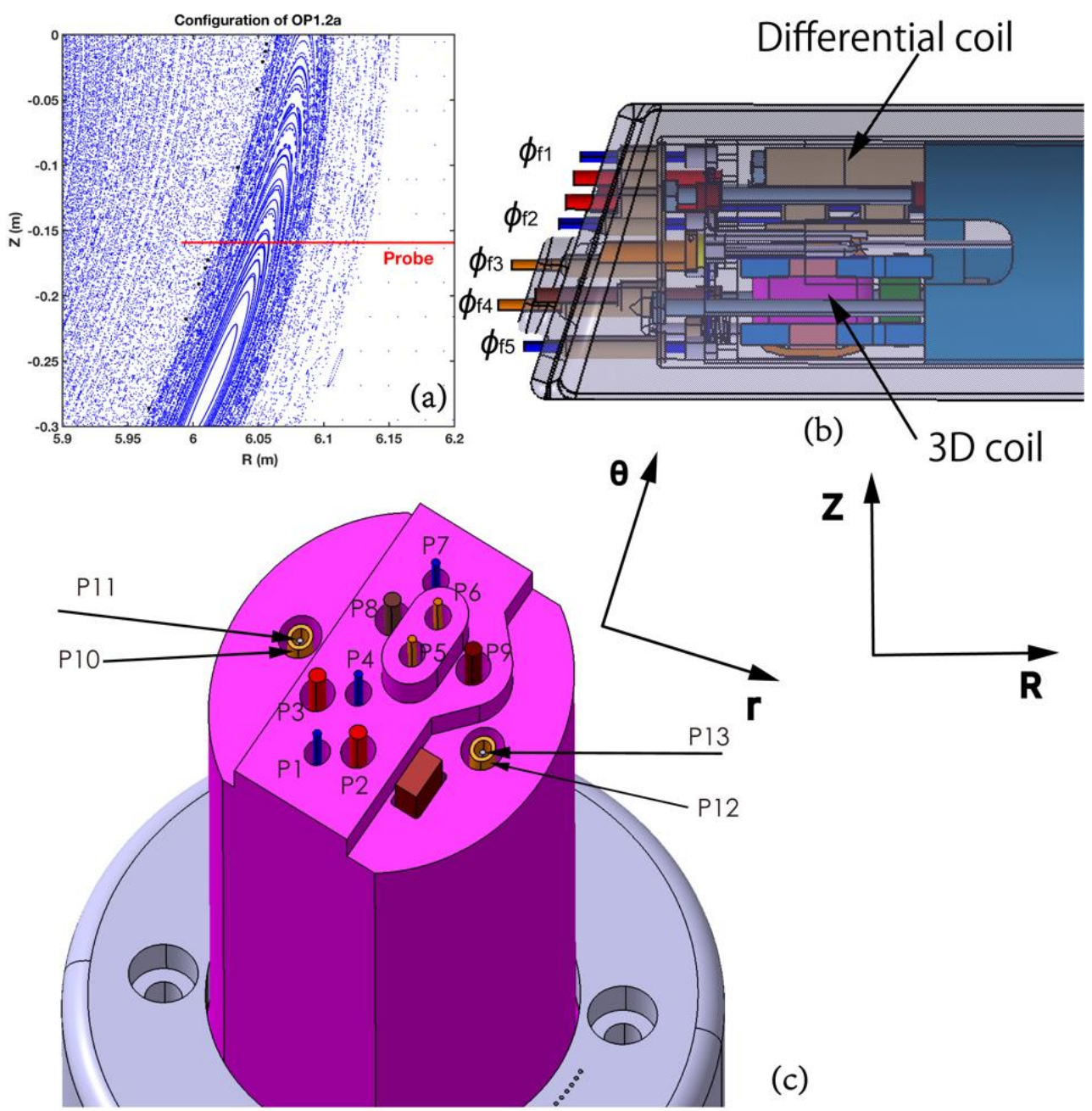

(c)

Figure 1. The sketch of the new combined probe used in OP1.2a. (a) A typical island divertor configuration in the poloidal plane of probe, and the red line denotes the probe path. (b) The projection of the probe head viewed along the toroidal direction. (c) The arrangement of all probe pins. 


\section{Experimental setup}

$\mathrm{W} 7-\mathrm{X}$ is a new optimized stellarator to accommodate a variety of 3D magnetic configurations, with a major radius of $5.5 \mathrm{~m}$ and a minor radius of about $0.5 \mathrm{~m}$ [26]. A multiple-purpose manipulator (MPM) has been developed and installed on W7$X$ in 2015, which is located under the outer midplane with $Z=-167 \mathrm{~mm}[27,28]$. The manipulator has a maximum plunge length of $35 \mathrm{~cm}$ for the fast movement, maximum acceleration of $30 \mathrm{~m} \mathrm{~s}^{-2}$, and a maximum speed of $2.5 \mathrm{~m} \mathrm{~s}^{-1}$. A new combined probe head, which consists of Langmuir probe pins, a Mach probe, an ion sensitive probe (ISP), a differential coil and a tri-axial pick-up coil, is developed and installed on the MPM in OP1.2a. With this new combined probe head, it is able to measure the edge plasma profiles $\left(T_{e}, n_{e}, \phi_{f}, M_{\|}\right)$, the variation of magnetic field, poloidal and radial turbulence structures. The sketch of the new combined probe is shown in Figure 1. A typical Poincaré plot of an island divertor configuration in vacuum case is shown in Figure 1(a), with the red line signifying the probe path which passes through the magnetic island in the SOL. As shown in Figure 1(b), the probe front surface is shaped to align on the local flux surface, consequently the probe pins are able to measure the plasma information on the same flux surface. Figure 1(c) shows the arrangement of probe pins, with pin $1,4,5,6$, and 7 measuring the floating potentials $\phi_{f 1}, \phi_{f 2}, \phi_{f 3}, \phi_{f 4}$ and $\phi_{f 5}$. Pin 2 and pin 3 are connected through a biasing voltage of $286 \mathrm{~V}$, forming a four-tip triple probe together with $\phi_{f 1}$ and $\phi_{f 2}$ to measure the electron density and temperature. The Mach probe consists of pin 8 and 9, giving the parallel flow velocity. There are two ion sensitive probes (ISP) on both sides of the middle stage, with pin 10 (12) as guard and pin 11 (13) as ion current collector. When both the guard and collector are biased with a sweeping voltage, it is able to measure the ion temperature [29-31]. The height and voltage difference between the guard and collector are two key parameters for the ISP. Besides the probe pins on the front surface, there is a tri-axial pick-up coil and a differential coil, while the magnetic variation along three directions $\left(B_{R}, B_{\varphi}, B_{Z}\right)$ can be obtained by the former coil and the local poloidal magnetic flux (i.e., local plasma current) is measured by the latter coil. Note that

Table 1. The parameters of the two configurations.

\begin{tabular}{llllll}
\hline Configuration & Shot No. & Non-planar coil (A) & Planar coil (A) & Trim coil (A) & $\begin{array}{l}\text { Toroidal } \\
\text { current (A) }\end{array}$ \\
\hline EJM+252 & 20171026.38 & $\begin{array}{l}13067,13066,13067, \\
13067,13067\end{array}$ & $-699,-699$ & $-84,17,96,41,-67$ & 1200 \\
Standard & & & & & \\
\hline KJM+252 & 20171017.56 & $12960,13213,13994$, & $-749,-749$ & $-95,1,95,59,-60$ & 700 \\
High mirror & & 12048,10920 & & & \\
\hline
\end{tabular}

the distance between the center of the differential coil and the front probe tip is $43 \mathrm{~mm}$, and this distance for the $3 \mathrm{D}$ coil is $39 \mathrm{~mm}$. This new combined probe head is designed to study the edge turbulence structure and transport. The turbulence poloidal structure can be derived from the combination of two pins among the pins $\phi_{f 1}, \phi_{f 2}, \phi_{f 5}$ (or the pins $\phi_{f 3}$ and $\left.\phi_{f 4}\right)$, and different poloidal distance for each combination. The radial structure is calculated by pin $\phi_{f 2}$ and $\phi_{f 3}$ (or pin $\phi_{f 4}$ and $\left.\phi_{f 5}\right)$. With this probe arrangement we can measure the radial heat and particle flux and the Reynold stress at the same time, and characterize the SOL turbulence structure and transport.

\section{Turbulence structures in the SOL with magnetic island}

Two magnetic configurations, EJM+252 and $\mathrm{KJM}+252$, have been used for most experimental programs in OP1.2a, where $\mathrm{EJM}+252$ is the standard configuration, and $\mathrm{KJM}+252$ is a high mirror configuration $[32,33]$. Two discharges with good measurements of the new combined probe have been selected to illustrate the SOL structures and turbulence behaviours in the two configurations, as listed in Table 1, including the current setting of the non-planar coils, planar coil and trim coils, as well as the toroidal plasma current during the plunge of the MPM. Figure 2 gives the plasma parameters of the two discharges. The heating power of electron cyclotron resonance heating $(\mathrm{ECRH})$ is about 1-2 MW, for the sake of making a deep plunge of MPM and measuring the profiles of the whole SOL magnetic island in a lower heat load environment. The plasma density is measured by a central channel of the Thomson scattering due to the lack of interferometer data in $\mathrm{KJM}+252$ configuration, around $1-3 \times 10^{19} \mathrm{~m}^{-3}$. The electron temperature from the Thomson scattering is about $3-4 \mathrm{keV}$, and the plasma energy is almost constant during the plunge of the probe, with the radial position of probe shown in Figure 2(f). Compared with the high-performance discharges, both shots here have lower heating power and line averaged density and stable plasma parameters during the plunge, which is beneficial to the characterization of the SOL properties. 


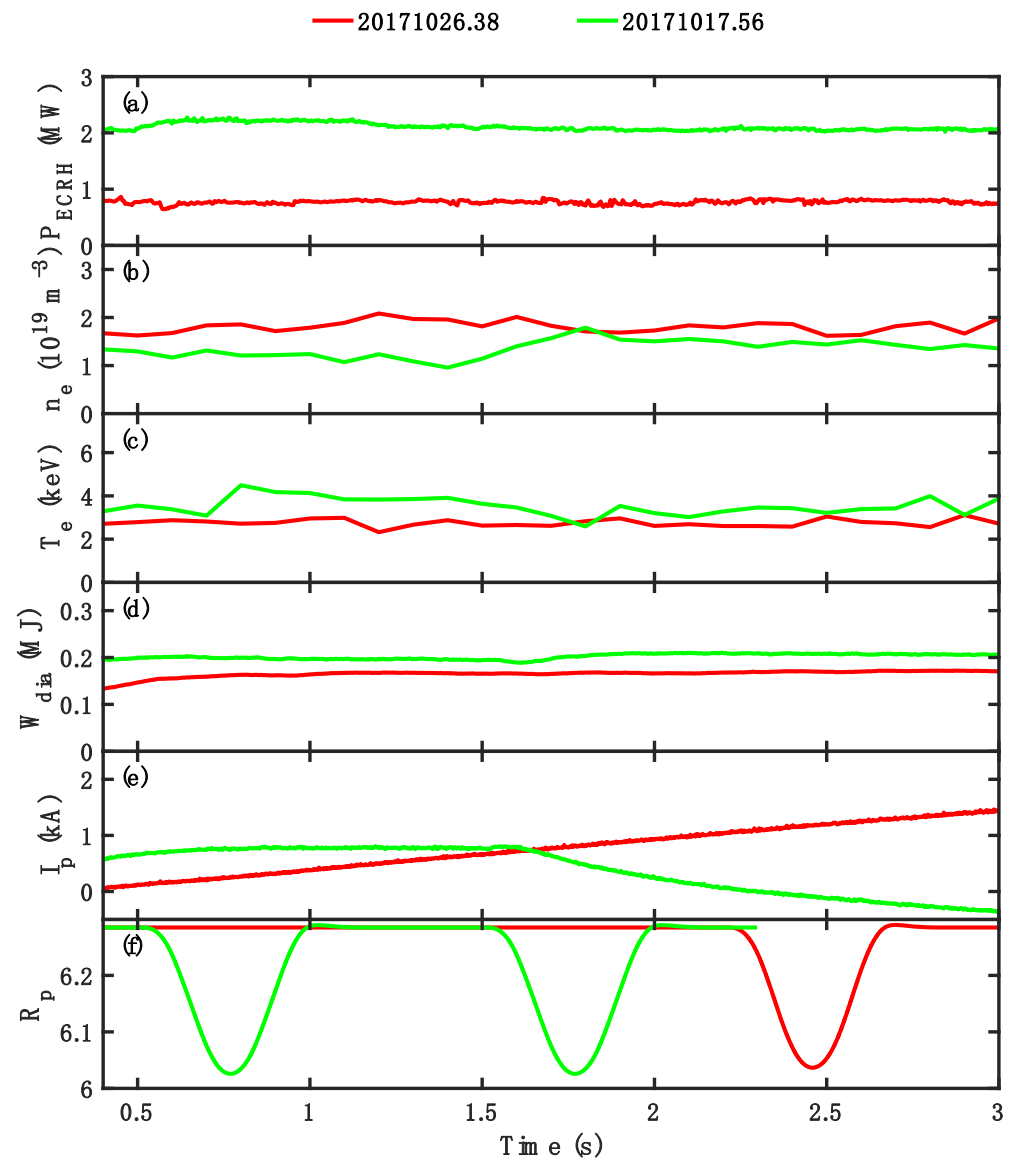

Figure 2. The plasma parameters for the two magnetic configurations in OP1.2a. (a) ECRH heating power. (b) Core electron density measured by Thomson scattering. (c) Core electron temperature measured by Thomson scattering. (d) Plasma energy. (e) Toroidal plasma current. (f) Radial position of the new combined probe.
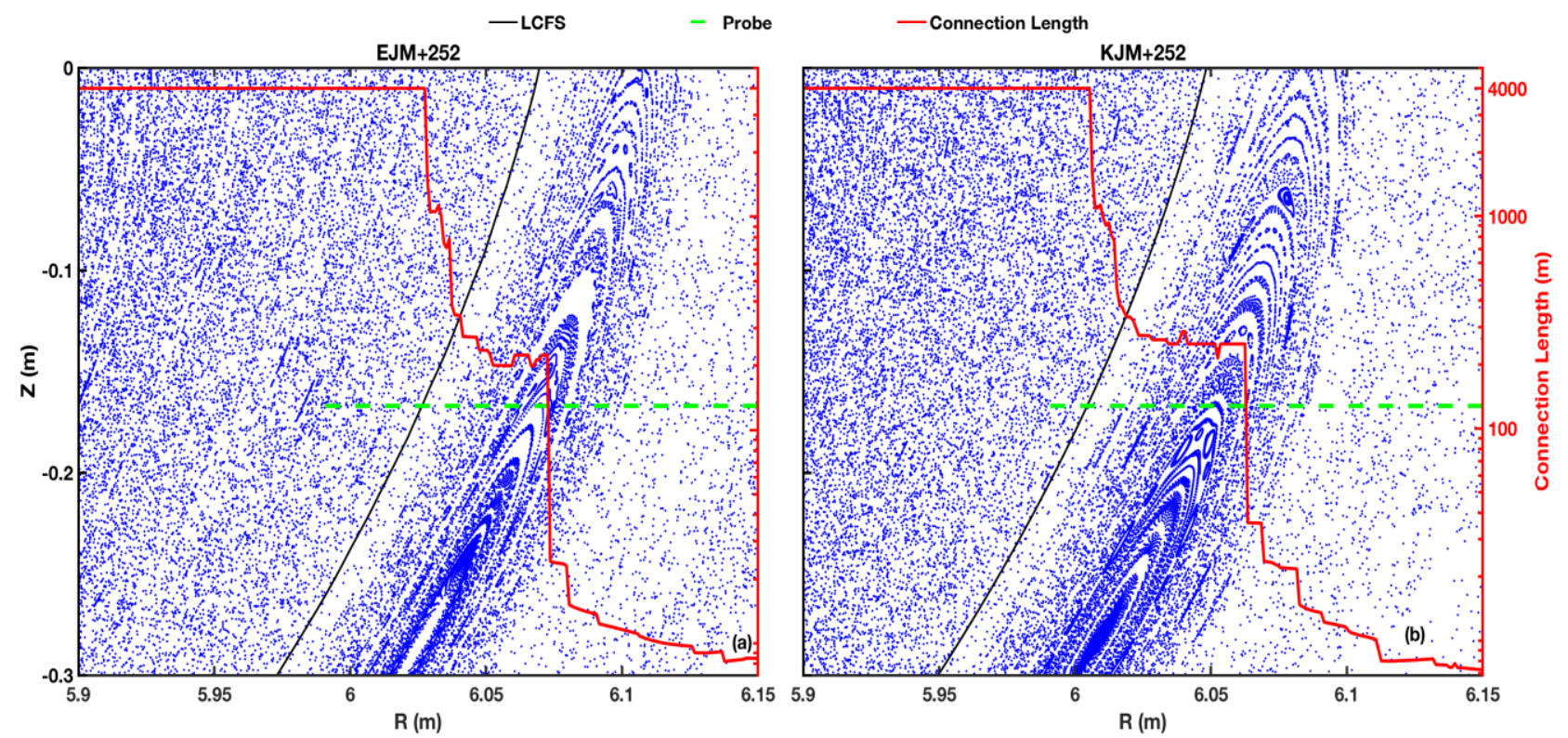

Figure 3. The Poincaré plot of the two configurations of OP1.2a on the poloidal plane of MPM. Panels from left to right are $\mathrm{EJM}+252$ and $\mathrm{KJM}+252$, respectively. 

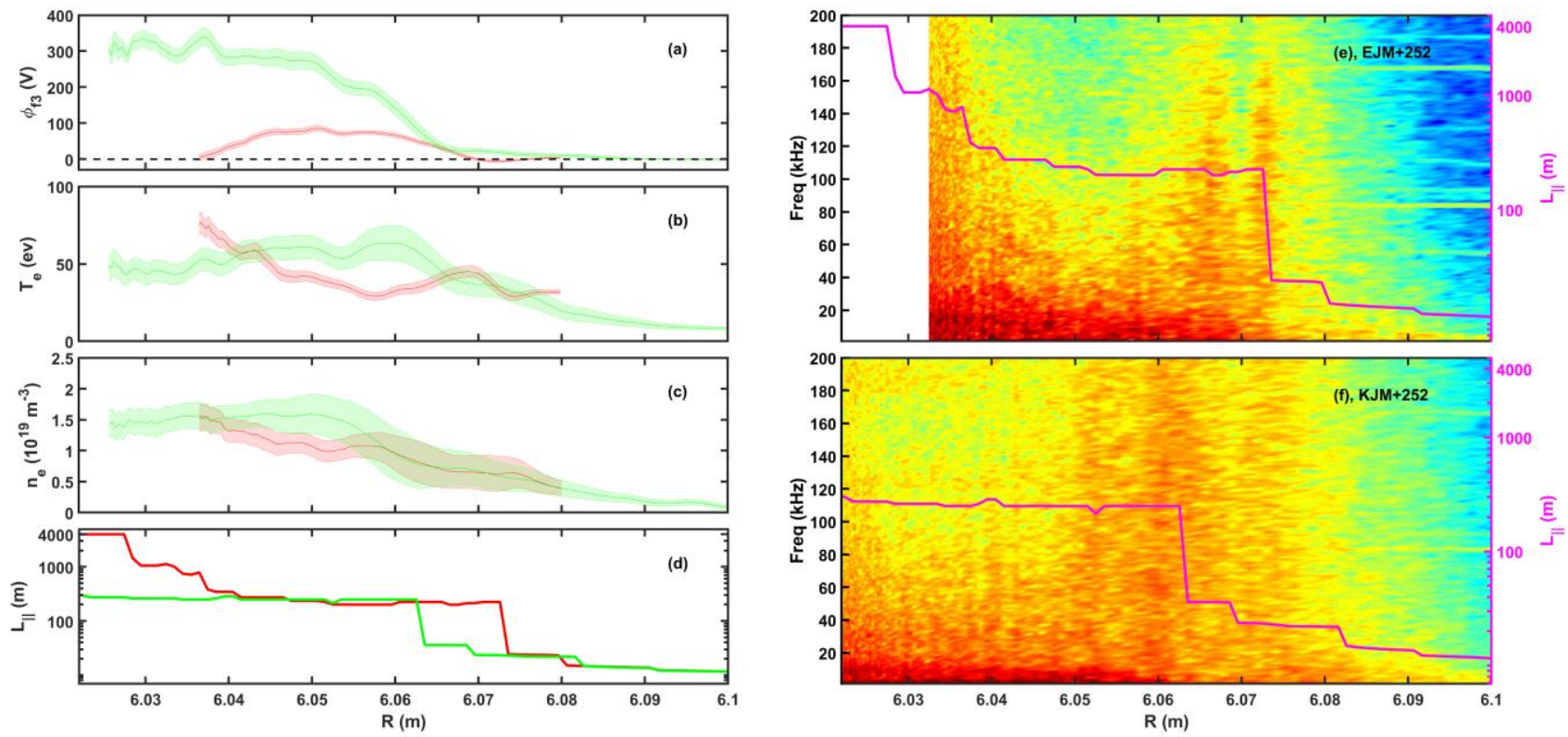

Figure 4. The plasma profiles measured by the new combined probe. (a-c) Floating potential, electron temperature, electron density; (d) Connection length; (e-g) The auto-correlation spectrum power density of $\phi_{f 3}$ for the two configurations. The shaded region in (b) and (c) denotes the standard deviation of the measurements.

Auto Correlation Power Spectrum, $\phi_{\mathrm{f} 3}$

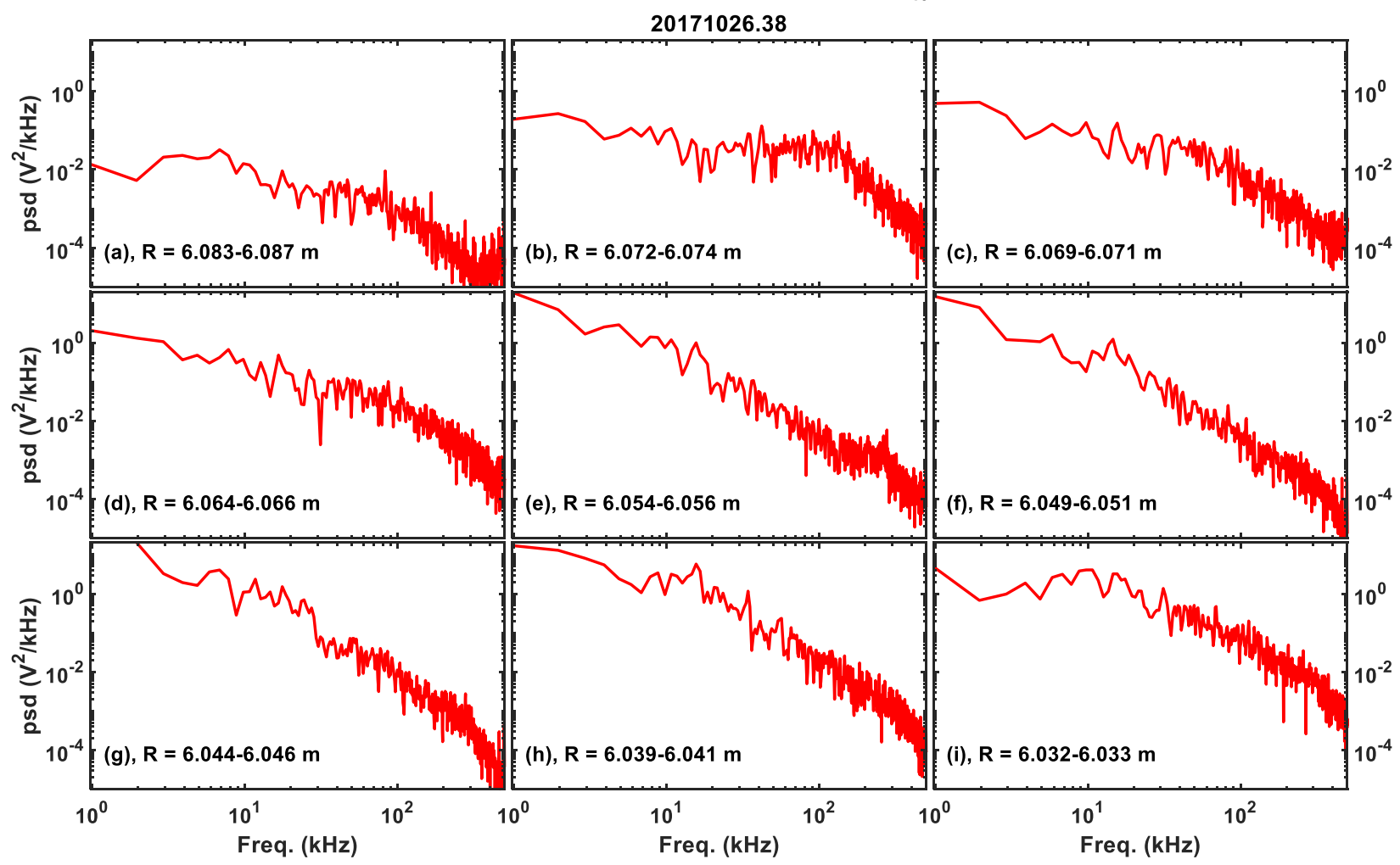

Figure 5. The auto-correlation power spectral density of floating potential $\phi_{f 3}$ at nine radial positions. 
The Poincaré plot of both configurations in the same poloidal projection as the MPM, and the connection length along the path of probe are calculated by a field line tracer with the real current settings in Table 1, as shown in Figure 3 [34-36]. It should be pointed out that the uncertainty of the magnetic equilibrium from field line tracer may be up to $1 \mathrm{~cm}$. When calculating the field line connection length, all the components of the first wall on W7-X in OP1.2a are included. For the standard configuration EJM+252, there is a broad magnetic island on the probe path, with an island width of $\sim 5 \mathrm{~cm}$ and the last closed flux surface (LCFS) located at $R=6.026 \mathrm{~m}$. In the far SOL, the connection length $L_{\|}$is below $15 \mathrm{~m}$ but increases sharply to over $200 \mathrm{~m}$ at the edge of island, then shows a flat region inside the island, then $L_{\|}$increases again between the inner edge of island and the LCFS, and finally $L_{\|}$ reaches the given threshold near the LCFS. The high mirror configuration $\mathrm{KJM}+252$ also exhibits similar variations for magnetic configuration and connection length, except that the island width is about $2 \mathrm{~cm}$ wider than EJM+252 configuration and the LCFS moves inward to about $R=6 \mathrm{~m}$. Moreover, there is a clear stochastic region between the LCFS and SOL island in both EJM+252 and KJM+252 configurations.

The typical SOL profiles measured by the new combined probe for both configurations are shown in Figure 4. The left panels display the floating potential of $\phi_{f 3}$, electron temperature $T_{e}$, electron density $n_{e}$ and the connection length along the path of the new combined probe, and the right panels display the auto-correlation power spectral density (APSD). The electron temperature and density are derived from the four-tip triple probe, with $T_{e}=\left[\phi_{+}-\left(\phi_{f 1}+\phi_{f 2}\right) /\right.$ $2] / \ln 2$ and $n_{e}=I_{s} /\left(0.49 e A_{e f f} \sqrt{T_{e} / m_{i}}\right)$, where $\phi_{+}$and $I_{s}$ are measured by the double-probe pin 3 and pin 2, respectively [37]. As shown in Figure 4 (a-c), the plasma profiles have strong dependence on the magnetic topology. In the $\mathrm{KJM}+252$ configuration, $T_{e}$ and $n_{e}$ present a synchronous increase with the connection length, and $n_{e}$ peaks around the island center along the probe path $(R \approx$ $6.052 \mathrm{~m})$. When the probe continues to plunge into the inner side of the island, both $T_{e}$ and $n_{e}$ decrease gradually to be saturated, but it is unable to form an in-out symmetric distribution about the island center. In the EJM+252 configuration, the island center along the probe path is located at $R \approx 6.069 \mathrm{~m}$. In this region, the floating potential becomes a negative valley, and $T_{e}$ exhibits a near symmetric peak. When $R<6.04 \mathrm{~m}$, the connection length increases quickly from $345 \mathrm{~m}$ to near $1000 \mathrm{~m}$ at the innermost point of the probe, and both $T_{e}$ and $n_{e}$ start to rise significantly. For both configurations, their APSDs of floating potential are raised to a much higher level when the connection lengths increase. In EJM+252 configuration, there is a broadband spectrum in the frequency range of $40-200 \mathrm{kHz}$ at the radial region of $R=$
$6.063-6.075 \mathrm{~m}$, but this broadband spectrum becomes weak or even disappears at the center of the island along the probe path (i.e., $6.068 \mathrm{~m} \leq R \leq 6.07 \mathrm{~m}$ ). As demonstrated by the two-dimension connection length in Figure 8, this narrow radial region has a slightly lower connection length in contrast to the adjacent radial region. When entering the radial region of $R<6.06 \mathrm{~m}$, the turbulence is dominated by low frequency fluctuations mainly below $30 \mathrm{kHz}$. Near the LCFS ( $R<6.038 \mathrm{~m}$ ), the fluctuations in high frequency are enhanced but still smaller than the fluctuations in a low frequency. The APSD of floating potential $\phi_{f 3}$ is shown in Figure 5 for 9 radial positions. In the far SOL with thin plasma, the fluctuation level of $\tilde{\phi}_{f 3}$ is relatively lower compared to the inner SOL region, as illustrated in Figure 5(a). The same as the observations in Figure 4(e), the broadband spectrum also appears when $R=6.072-6.074 \mathrm{~m}$ and $R=6.064-$ $6.066 \mathrm{~m}$, as displayed in Figure 5(b) and (d), with the fast decay of the ASPD starting from $110-140 \mathrm{kHz}$. The mitigation of the APSD is also observed in Figure 5 (c) at the radial location of $R=6.069-6.071 \mathrm{~m}$, with the APSD starting to decrease dramatically at $60 \mathrm{kHz}$. When the probe plunging deeper inside, the APSD in the frequency range of $40-200 \mathrm{kHz}$ is very low and drops quickly with frequency, simultaneously the APSD in the low frequency range $(10-30 \mathrm{kHz})$ increases significantly, as presented in Figure 5(f)-(i).

The KJM+252 configuration also presents similar APSD radial evolution as the standard configuration, with the island center on the probe path changing to $R=6.052 \mathrm{~m}$ and the broadband spectrum becoming weak in this region. Because the innermost point of the probe is $2 \mathrm{~cm}$ away from the LCFS, the significant rise of the high frequency fluctuations near the LCFS is not seen in Figure 4(f). It should be pointed out that in both discharges a very large positive floating potential (several hundred volt) has been observed in the island divertor topology, indicating a strong imbalance of ions and electrons in the upstream plasmas. The resonant magnetic perturbation (RMP) induced by external coils in tokamaks has been demonstrated to modify the edge magnetic topology and plasma profiles [38-40]. During the dynamic ergodic divertor (DED) $m / n=6 / 2$ (with $m / n$ as the poloidal/toroidal mode number) phase in TEXTOR, the SOL electron temperature and density have been reduced, but the floating potential turns to positive value and reaches $+30 \mathrm{~V}$ inside the ergodic zone where the connection length of the stochastic field line is longer than the Kolmogorov length, resulting in a large positive radial electric field in this region [40]. The interpretation for the positive floating potential is that the electrons move faster than massive ions along the field lines to the wall in the plasma with open stochastic field lines. It is similar with the probe measurements in W7-X, i.e., large positive floating potential in the open stochastic field lines. 


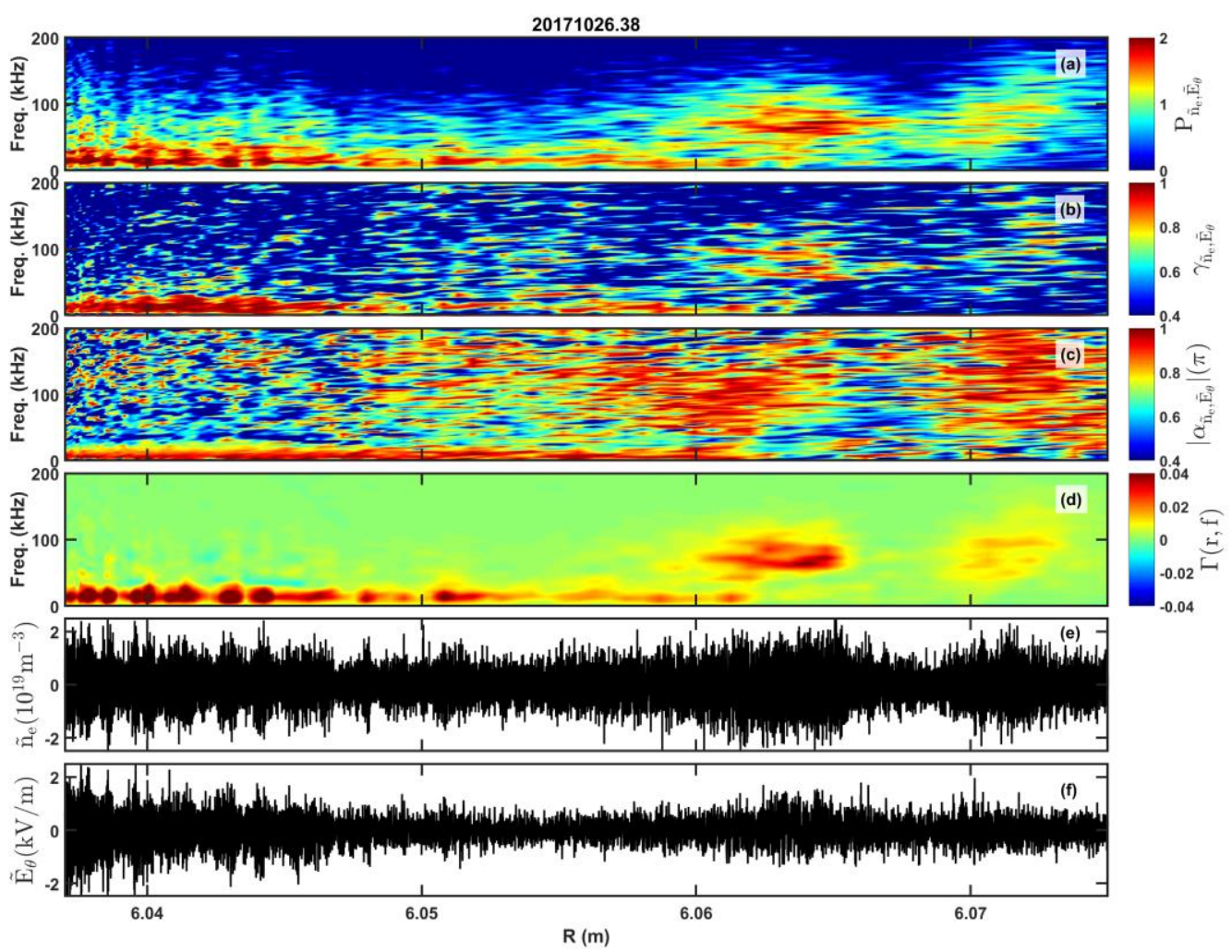

Figure 6. The cross-correlation between $\tilde{n}_{e}$ and $\tilde{E}_{\theta}$. (a) Cross-correlation power spectral density $P_{\tilde{n}_{e} \tilde{E}_{\theta}}$; (b) Cross-Correlation coefficient $\gamma_{\tilde{n}_{e} \tilde{E}_{\theta}}$; (c) Cross-Correlation phase $\alpha_{\tilde{n}_{e} \tilde{E}_{\theta}}$; (d) Turbulent particle flux.; (e) and (f) the fluctuations of $n_{e}$ and $E_{\theta}$ between the frequency range of $1-400 \mathrm{kHz}$, respectively.

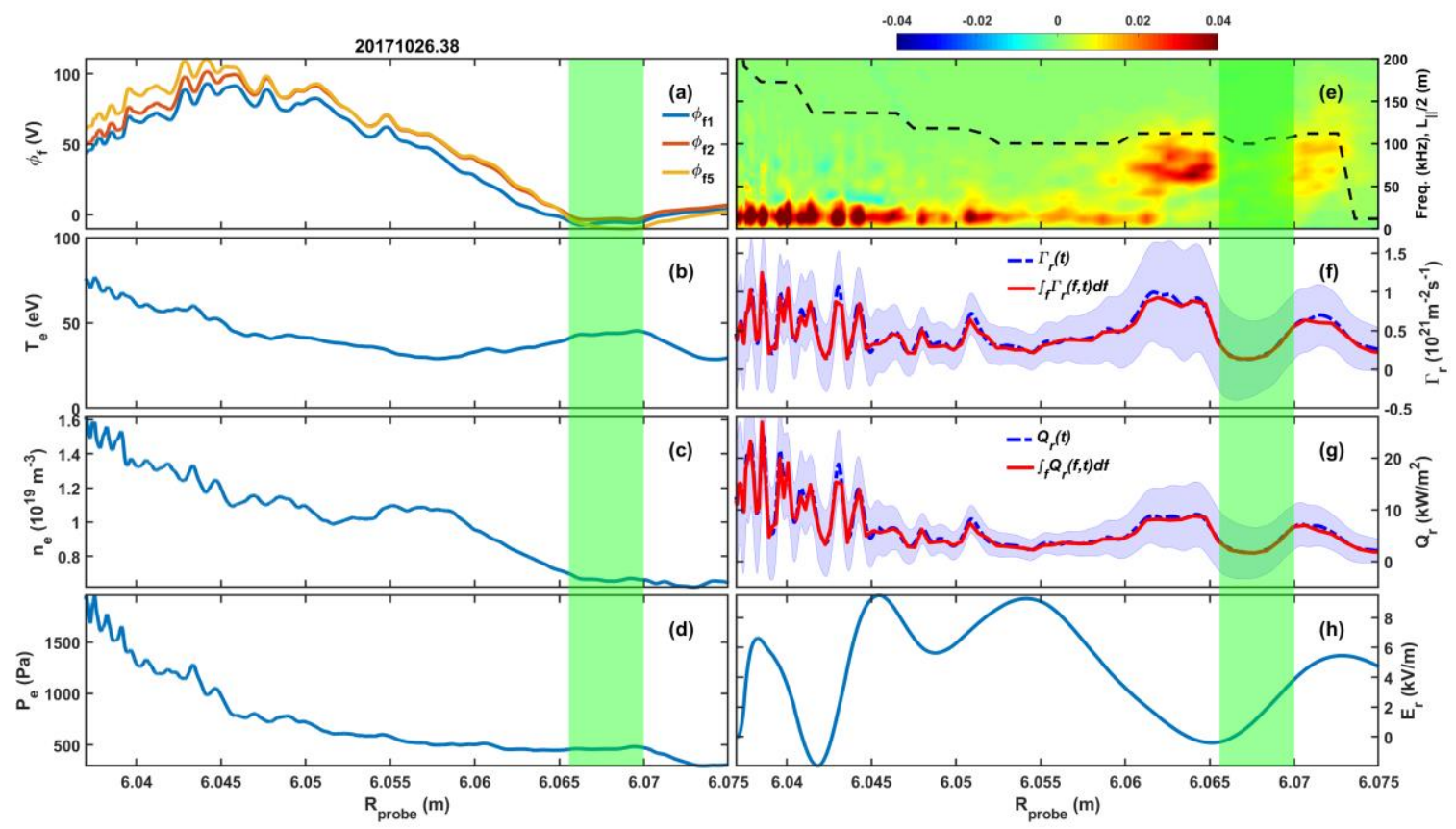

Figure 7. The turbulent radial heat flux, particle flux and plasma profiles. (a-d) floating potential, electron temperature, electron density and electron pressure. (e) The distribution of the turbulent radial particle flux in frequency space and the normalized connection length (dashed line). The green rectangle signifies a region with flat electron pressure and low radial heat flux. Positive value means the particle flux is directed outward. (f) The radial turbulent particle flux. (g) The radial turbulent heat flux. (h) Radial electric field. The shaded region in (f) and (g) denotes the standard deviation. 


\section{Turbulence transport in standard configuration}

The SOL radial heat and particle transport in the standard configuration and high mirror configuration of OP1.2a will be presented in this manuscript. In the standard configuration, the smallest distance between the probe and the LCFS is $6 \mathrm{~mm}$, which is much closer than in the $\mathrm{KJM}+252$ configuration. As a result, the EJM+252 case will be analyzed in detail to illustrate the transport characteristics induced by turbulence. The radial particle flux and heat flux can be derived from the four-tip triple probe, pin 1-4, as shown in Figure 1. Usually, the particle flux and heat flux driven by turbulence is calculated from the fluctuations of density, temperature and electric field, as shown in the following formulas:

$\Gamma_{e}=\left\langle\tilde{n}_{e} \tilde{V}_{r}\right\rangle=\frac{\left\langle\tilde{n}_{e} \tilde{E}_{\theta}\right\rangle}{B_{\varphi}}$

$\mathrm{Q}_{e}=\frac{3}{2}\left\langle\widetilde{P}_{e} \tilde{V}_{r}\right\rangle=\frac{3 T_{e}\left\langle\tilde{n}_{e} \tilde{E}_{\theta}\right\rangle}{2 B_{\varphi}}+\frac{3 n_{e}\left\langle\tilde{T}_{e} \tilde{E}_{\theta}\right\rangle}{2 B_{\varphi}}$

Where the tilde on the top denotes the fluctuation of the signal, the angle bracket denotes the ensemble average, and $B_{\varphi}$ is the toroidal magnetic field, $E_{\theta}=\left(\phi_{f 2}-\phi_{f 1}\right) /$ $d$. In addition, the particle and heat fluxes in the frequency space can be obtained by the cross-correlation power between fluctuations of density and electric field (or temperature and electric field) as shown in the following formulas:

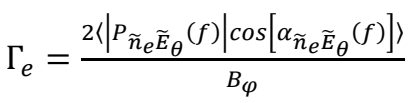

$\mathrm{Q}_{e}(f)=\frac{3}{2} T_{e} \Gamma_{e}(f)+\frac{3 n_{e}\left\langle\left|P_{\widetilde{T}_{e} \widetilde{E}_{\theta}}(f)\right| \cos \left[\alpha_{\widetilde{T}_{e} \widetilde{E}_{\theta}}(f)\right]\right\rangle}{B_{\varphi}}$

$P_{\tilde{n}_{e} \tilde{E}_{\theta}}(f)$ is the cross power spectrum of fluctuations of electron density and the fluctuations of the poloidal electric field, $\alpha_{\tilde{n}_{e} \tilde{E}_{\theta}}(f)$ is the cross-phase angle between these two parameters, and $B_{\varphi}$ is the toroidal magnetic field. It is assumed that the difference of the $\tilde{T}_{e}$ at the two poloidal positions of $\phi_{f 1}$ and $\phi_{f 2}$ is much smaller than the fluctuation amplitude of $\phi_{f 2}-\phi_{f 1}$.

The radial turbulent particle flux in the frequency space $\Gamma_{r}(f, t)$ derived from equation (3) is shown in Figure 6(d) and Figure 7(e). The cross-correlation between $\tilde{n}_{e}$ and $\tilde{E}_{\theta}$ is shown detailed in Figure 6 (a-c), i.e., cross-correlation power spectral density (CPSD) $P_{\tilde{n}_{e} \tilde{E}_{\theta}}$, cross-correlation coefficient $\gamma_{\tilde{n}_{e} \tilde{E}_{\theta}}$ and cross-correlation phase $\alpha_{\tilde{n}_{e} \tilde{E}_{\theta}}$. In consistent with the turbulence characteristics in Figure 4 and Figure 5, in the broadband turbulence dominant region ( $R=6.072-$ $6.074 \mathrm{~m}$ and $R=6.064-6.066 \mathrm{~m}$ ), there is strong CPSD $P_{\tilde{n}_{e} \tilde{E}_{\theta}}$ in the frequency range of $40-120 \mathrm{kHz}$, accompanied by high $\gamma_{\tilde{n}_{e} \tilde{E}_{\theta}}$ and $\left|\alpha_{\tilde{n}_{e} \tilde{E}_{\theta}}\right|>0.8 \pi$. In consequence, a large radial particle flux induced by this broadband fluctuations is observed in Figure 6 (d). In the radial region of $6.066 \mathrm{~m} \leq$ $R \leq 6.07 \mathrm{~m}$, the fluctuations of $n_{e}$ and $E_{\theta}$ between the frequency range of $1-400 \mathrm{kHz}$ are mitigated significantly, resulting in the low $P_{\tilde{n}_{e} \tilde{E}_{\theta}}, \gamma_{\tilde{n}_{e} \tilde{E}_{\theta}},\left|\alpha_{\tilde{n}_{e} \tilde{E}_{\theta}}\right|$ and turbulent particle flux in this region. When $R<6.06 \mathrm{~m}$, the CPSD $P_{\tilde{n}_{e} \tilde{E}_{\theta}}$ of the broadband turbulence decreases sharply to a low level. At the same time, the low frequency $(10-30 \mathrm{kHz})$ turbulence contributes to large $P_{\tilde{n}_{e} \tilde{E}_{\theta}}, \gamma_{\tilde{n}_{e} \tilde{E}_{\theta}}$ and $\left|\alpha_{\tilde{n}_{e} \tilde{E}_{\theta}}\right|=$ $0.8 \pi-\pi$, especially when $R<6.045 \mathrm{~m}$, leading to a low frequency dominant radial particle flux. Although both $\tilde{n}_{e}$ and $\tilde{E}_{\theta}$ reveal different fluctuation patterns in the broadband turbulence region and the low frequency turbulence region, the emergence of large turbulent particle flux is synchronous with the strong fluctuation in $n_{e}$ and $E_{\theta}$. In addition, it is an overlap between the high frequency broadband turbulence and the low frequency turbulence in the radial range of $R=6.058$ $6.064 \mathrm{~m}$. It should be noted that the cross-correlation phase $\alpha_{\tilde{n}_{e} \tilde{E}_{\theta}}$ is negative and around -1 to -0.8 for the above two types of radial turbulent transport, i.e., the fluctuations in electron density lags behind the fluctuations in poloidal electric field.

Typical radial turbulent transport behaviours are illustrated in Figure 7. The electron pressure is displayed in Figure 7(d), which has an extremely flat profile in the radial region of $R=$ $6.066-6.07 \mathrm{~m}$, as marked in the green rectangle. The floating potentials in Figure 7(a) exhibit a negative basin in this region. Besides, both $T_{e}$ and $n_{e}$ have slow variations, and the parallel Mach number is $M_{\|} \sim 0$ within this radial region. The radial electric field, derived from $E_{r}=-d\left(\phi_{f}+\right.$ $\left.2.8 T_{e}\right) / d R$, continues to decrease with decreasing $R$ within the green rectangle. According to the Poincaré plot of the standard configuration in Figure 3, the magnetic island center is mainly located in this green rectangle, characterized by flat profiles of $T_{e}, n_{e}, p_{e}, M_{\|}$and a slightly negative $\phi_{f}$.

In Figure $7(\mathrm{e}), \Gamma_{r}(f, t)$ reveals a strong dependence on magnetic topology, i.e., it is dominated by high frequency fluctuations in the outer SOL but by low frequency turbulence in the inner SOL. In the radial region of $R=6.06-$ $6.073 \mathrm{~m}$, strong outward particle flux is driven by a broadband turbulence within the frequency range of 40-120 $\mathrm{kHz}$, which is consistent with the large APSD of the floating potential in this region, as illustrated in Figure 4(e). However, inside the green rectangle the particle flux is very weak, which also agrees well with the APSD of Figure 4(e). When the probe goes to the near SOL, $\Gamma_{r}(f, t)$ decreases significantly when $R<6.06 \mathrm{~m}$, and the particle transport is dominated by low frequency turbulence $(10-20 \mathrm{kHz})$. In this near SOL region, the turbulence transport reflects some intermittent structures, especially when getting close to the LCFS. It should be pointed out that the intermittent event is generally 
associate to a temporal bursty behavior. In this article the word 'intermittent' is used to signify the bursty transport structure in Figure 7, which could be associate to the spatial inhomogeneity. Figure 7(f) presents the radial turbulent particle flux, with the blue line derived from equation (1) and the red dashed line calculated from $\int \Gamma_{r}(f, t) d f$. Note that $\Gamma_{r}$ from equation (1) has been smoothed to signify the mean particle flux. The results demonstrate that both the time domain analysis and frequency domain analysis are in good agreement with each other. In the whole SOL region, the particle flux driven by turbulence is directed outward, and exhibits a minimum value in the green rectangle region, i.e., the turbulence transport is mitigated in this region. In accordance with Figure 7(e), the radial particle flux in the whole frequency region inside the green rectangle is suppressed, while on both sides of the rectangle there are two peaks located at $R=6.06-6.065 \mathrm{~m}$ and $R=6.07-$ $6.073 \mathrm{~m}$, and the peak value of the mean $\Gamma_{r}$ is as large as $10^{21}$ $\mathrm{m}^{-2} \mathrm{~s}^{-1}$. It should be pointed out that this mean radial particle flux can be up to several $10^{22} \mathrm{~m}^{-2} \mathrm{~s}^{-1}$ in high heating power and plasma density discharges. Within the radial region of $R=$ $6.045-6.058 \mathrm{~m}, \Gamma_{r}$ drops to $\sim 0.4 \times 10^{21} \mathrm{~m}^{-2} \mathrm{~s}^{-1}$. When $R<$ $6.045 \mathrm{~m}$, the base line of $\Gamma_{r}$ increases gradually with decreasing $R$. There are some spikes on the profile of particle flux in this region, which are contributed by the low frequency fluctuations with a central frequency $\sim 15 \mathrm{kHz}$. Figure 7 shows the radial turbulent heat fluxes calculated by both equation (2) and (4), which reveal similar radial variations as the radial particle flux with maximum $Q_{r}$ about $10 \mathrm{kWm}^{-2}$ in the broadband turbulence region. To sum up, a significant change can be found near $R=6.06 \mathrm{~m}$, i.e., the dominant frequency for radial transport switches from the broadband spectrum (40$120 \mathrm{kHz})$ to low frequency turbulence $(10-20 \mathrm{kHz})$.

The two-dimension magnetic field connection length derived from the field line tracer which involves all the components of first wall in OP1.2a is shown in Figure 8. Three dot lines are added in the figure to illustrate the radial position of $R=6.06$, 6.065 and $6.07 \mathrm{~m}$. Along the probe path, there are two light blue regions located at $R=6.052-6.06 \mathrm{~m}$ and $R=$ $6.066-6.07 \mathrm{~m}$, i.e., shorter connection length in these two regions. As measured in Figure 7, in these two light blue regions, the radial turbulent heat and particle fluxes reduce to very low levels. While the two broadband spectrum dominant regions are also consistent with the light green regions having longer connection lengths. Connection length increases to about $300 \mathrm{~m}$ when $R<6.047 \mathrm{~m}$, and meanwhile the intermittent events dominate the radial transport in Figure 7(e). Therefore, both the turbulence structure and the radial transport are sensitive to the magnetic topology in the SOL of W7-X.

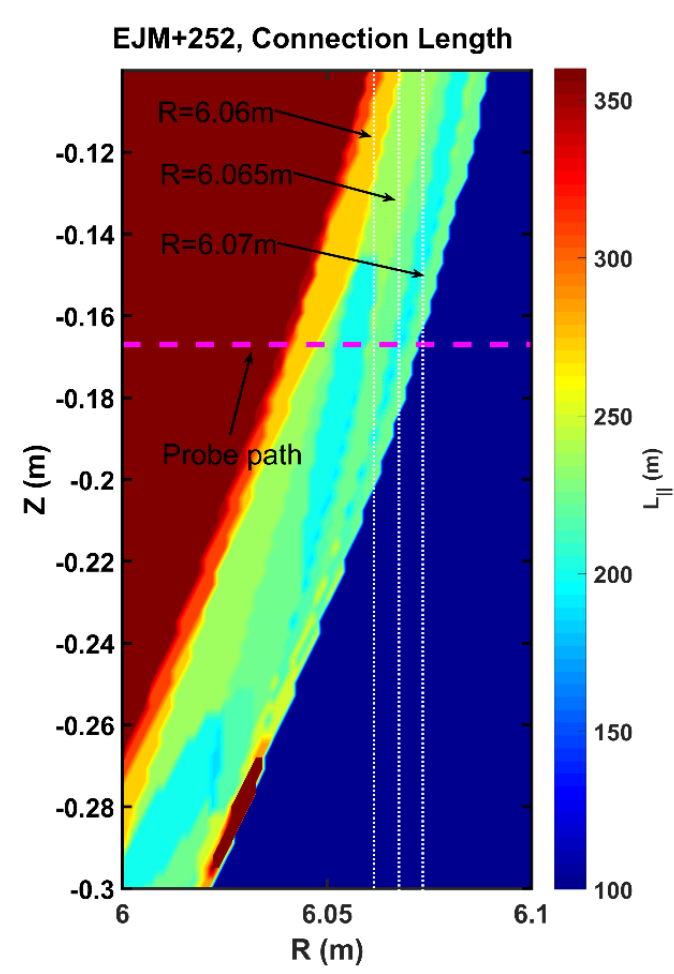

Figure 8. The two-dimension connection length on the poloidal plane of MPM for the EJM+252 configuration.

\subsection{Turbulence transport driven by broadband turbulence}

In the radial region of $R=6.06-6.065 \mathrm{~m}$ and $R=$ $6.07-6.073 \mathrm{~m}$, the radial turbulent heat and particle transport is driven by a broadband spectrum with frequency from 40 to $120 \mathrm{kHz}$. Between these two radial regions the radial transport is mitigated to a relatively low level, as shown in the green rectangle of Figure 7(e). Meanwhile, inside the rectangle, there is a flat pressure profile and negative shear of electric field $\partial E_{r} / \partial R$, which could contribute to suppress the broadband turbulence and reduce the radial transport [41-43]. It is obvious that a steep density gradient exists in the radial region of $6.059 \leq R \leq 6.065 \mathrm{~m}$, as illustrated in Figure 7(c), in which the large particle flux induced by the broadband spectrum is also located, indicating that this turbulence transport could be driven by a density-gradient related turbulence. Figure 9 displays the APSD of ion saturation current and the CPSD between two radially separated floating potential pins $\phi_{f 2}$ and $\phi_{f 3}$, with $R=6.064 \mathrm{~m}$ for the broadband spectrum dominant region and $R=6.037 \mathrm{~m}$ for the low frequency dominant region. In the APSD of ion saturation current, a peak appearing at $f \sim 70 \mathrm{kHz}$ is one magnitude larger than the APSD in low frequency region. The spectral density is fitted by the spectral power decay function 
$S \propto f^{\alpha}$ in some interesting frequency ranges. In Figure 9(a), the APSD decreases quickly on both sides of the peak, with a decay factor $|\alpha|>3.8$ for both EJM+252 and KJM+252 configurations. This demonstrates that the fluctuations of particle flux are primarily contributed by the broadband spectrum centered at $70 \mathrm{kHz}$ for the EJM+252 configuration. The radial cross-correlation between $\phi_{f 2}$ and $\phi_{f 3}$ in Figure 9(c) displays a flat CPSD from 40 to $70 \mathrm{kHz}$, and then decreases with a factor of $\alpha=-2.46$ for the EJM+252 configuration, signifying the radial structure of the broadband spectrum. Compared with the standard configuration, the $\mathrm{KJM}+252$ configuration has a much wider flat region of CPSD and a smaller power decay factor of $\alpha=-1.37$, indicating that the broadband spectrum has a broader frequency distribution.

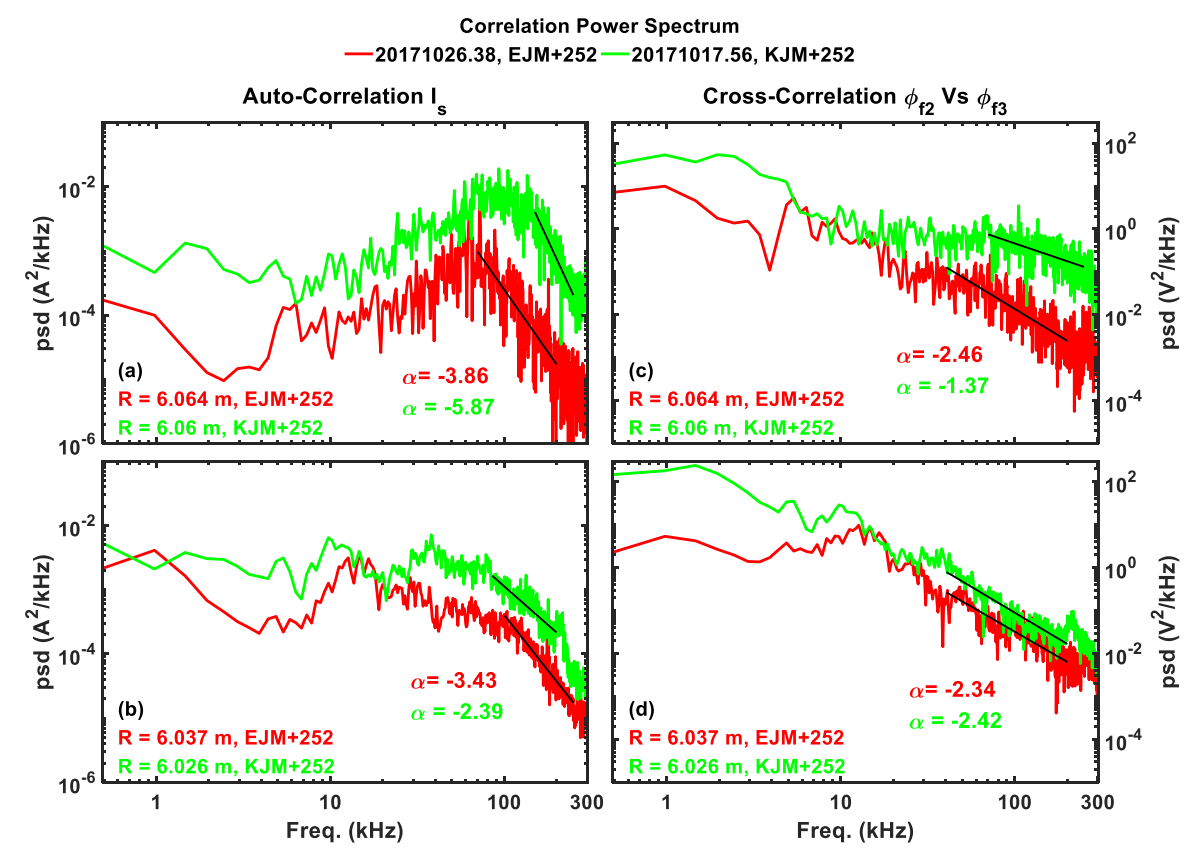

Figure 9. The APSD of the ion saturation current (left panels) and the cross-correlation spectral density (CPSD) between two radially separated floating potential pins (right panels), for both EJM+252 and KJM+252 configurations.
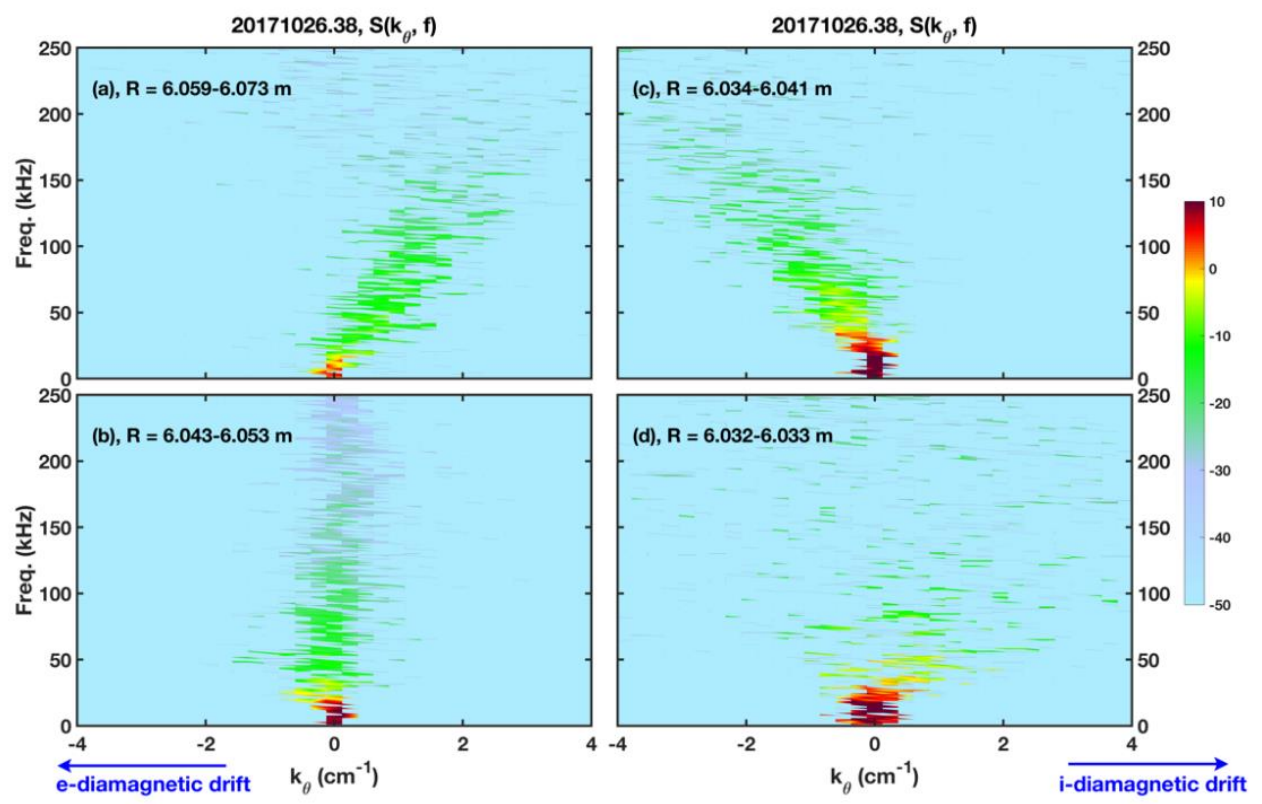

Figure 10. The poloidal cross-correlation power spectral density $S\left(k_{\theta}, f\right)$ at four radial positions along the probe path for EJM+252 configuration. 
In order to identify the propagation feature, a normalized poloidal cross-correlation power spectral density $S\left(k_{\theta}, f\right)$ has been calculated through the two-point cross-correlation technique and shown in Figure 10 [40, 44, 45]. Note that two floating potential pins $\phi_{f 3}$ and $\phi_{f 4}$ are selected to present the statistical property of the poloidal turbulence structure, because they are positioned $4 \mathrm{~mm}$ closer than the four-tip probes. The broadband spectrum in the far SOL is extremely clear in Figure 10 (a), located in the frequency range $f=40$ $120 \mathrm{kHz}$ and wavenumber $k_{\theta}=0.3-2.5 \mathrm{~cm}^{-1}$. Estimated from the slope of $S\left(k_{\theta}, f\right)$, the phase velocity of the broadband turbulence is $V_{\text {phase }}=2 \pi \Delta f / \Delta k_{\theta} \approx 4.6 \mathrm{~km} / \mathrm{s}$, propagating in the ion diamagnetic drift direction in the laboratory frame. The radial electric field in Figure $7(\mathrm{~h})$ is $0.5-5 \mathrm{kV} / \mathrm{m}$ within the broadband turbulence region, in consequence the poloidal electric drift velocity is $V_{E \times B}=E_{r} / B_{\varphi}=0.5 / 2.2-5 / 2.2=$ $0.23-2.27 \mathrm{~km} / \mathrm{s}$ along the ion diamagnetic drift direction with toroidal magnetic field in anti-clockwise direction viewed from top. As a result, the propagation velocity of the broadband turbulence in the plasma frame is $V_{\text {phase }}-$ $V_{E \times B} \approx 2.3-4.4 \mathrm{~km} / \mathrm{s}$ along the ion diamagnetic drift direction. When $R<6.06 \mathrm{~m}$ the $S\left(k_{\theta}, f\right)$ reveals three different spectral patterns, which will be discussed in the next section. The ion sound Larmor radius derived from $\rho_{s}=$ $\sqrt{2 T_{e} m_{i}} / e B$ is about $0.7-0.8 \mathrm{~mm}$ in the radial region of the broadband spectrum, which gives the local turbulence parameter $k_{\theta} \rho_{s}=1 \mathrm{~cm}^{-1} * \rho_{s} \approx 0.07-0.08$ at the center frequency $(70 \mathrm{kHz})$ of the broadband. The ion diamagnetic frequency obtained by $\omega_{* i}=-\left(k_{\theta} c\right) /(e B n) d p / d r$ is about $20-140 \mathrm{rad} / \mathrm{kHz}$, and $f_{* i}=\omega_{* i} / 2 \pi=5-20 \mathrm{kHz}$ in the broadband region, where $T_{i}=T_{e}$ and $n_{i}=n_{e}$ are assumed $[46,47]$. Considering the electric drift frequency, $f_{E \times B}=$ $k_{\theta} V_{E \times B} / 2 \pi \approx 40 \mathrm{kHz}$ for the broadband spectrum, and the observed frequency in laboratory frame $f_{l a b}=f_{* i}+f_{E \times B}$ can be as large as $60 \mathrm{kHz}$, which is located near the central frequency of the broadband spectrum [48].

Figure 11(a) gives the relative fluctuation levels of electron density and electron temperature with the formula $\delta x / x=$ $\sqrt{\sum(x(t)-\bar{x}) / N} / \bar{x}$, where the number of signal point $N$ is set to $2048(\sim 1 \mathrm{~ms})$. In the broadband spectrum region, $\delta n_{e} / n_{e} \approx 1.2, \delta T_{e} / T_{e} \approx 0.1$, and their ratio $\left(\delta n_{e} / n_{e}\right) /$ $\left(\delta T_{e} / T_{e}\right) \approx 10$, i.e., the relative fluctuations from density are much larger than that from temperature. A similar relationship between fluctuations of $n_{e}$ and $T_{e}$ was measured by the Langmuir probes in the SOL of the TEXT tokamak, with $\delta n_{e} / n_{e}=(0.3-0.4) \delta T_{e} / T_{e}$, and the ion saturation current and floating potential also exhibit a broadband structure [49]. The normalized electron density and temperature gradient scale lengths are shown in Figure 11(b), with $R / L_{n_{e}}=$ $\left|\left(R / n_{e}\right) d n_{e} / d r\right|$ and $R / L_{T_{e}}=\left|\left(R / T_{e}\right) d T_{e} / d r\right|$, where $R$ is the major radius. Both parameters have large values at the radial region of $R=6.06-6.065 \mathrm{~m}$ where the radial transport is enhanced by the broadband turbulence. From the equation of $\Gamma_{e}=-D_{e} \partial n_{e} / \partial r$, the radial particle diffusion coefficient is estimated and shown in Figure 11(c), with $D_{e} \approx$ $1-2.3 \mathrm{~m}^{2} \mathrm{~s}^{-1}$ for the broadband region $6.06 \mathrm{~m} \leq R \leq$ $6.065 \mathrm{~m}$.

In brief, the broadband turbulence is centered at $70 \mathrm{kHz}$, propagates in the ion diamagnetic drift direction in both the Laboratory frame and the plasma frame, with $k_{\theta} \rho_{s}=0.7-$ $0.8,\left(\delta n_{e} / n_{e}\right) /\left(\delta T_{e} / T_{e}\right) \approx 10$ and very large normalized gradient scale lengths $R / L_{n_{e}}$ and $R / L_{T_{e}}$. The effective particle diffusion coefficient induced by turbulence in this broadband region is about $D_{e}=1-2.3 \mathrm{~m}^{2} \mathrm{~s}^{-1}$. Although the characteristics of the turbulence mode agree with the ITG mode in some aspects, such as the propagation direction, $k_{\theta} \rho_{s}$ and gradient scale length, it is still difficult to identify the mode of this broadband turbulence, because usually ITG has larger an ion temperature relative fluctuation level than the density relative fluctuation level. It will be nicer to leave the identification of this mode to future work with simulations.

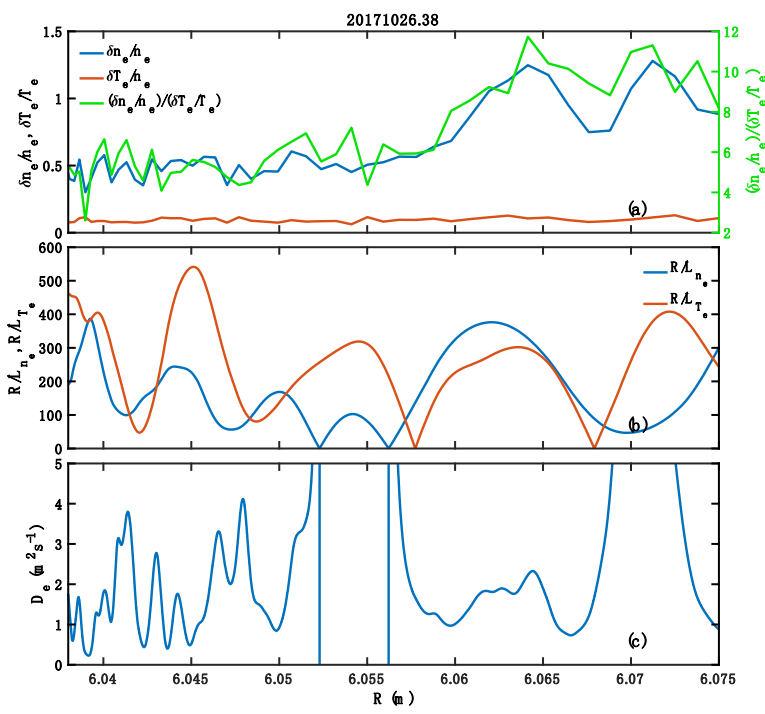

Figure 11. (a) Relative fluctuation levels of the electron density and temperature, and their ratio $\left(\delta n_{e} / n_{e}\right) /\left(\delta T_{e} / T_{e}\right)$. (b) Normalized gradient scale length of electron density and temperature. (c) Electron diffusion coefficient induced by turbulence. 

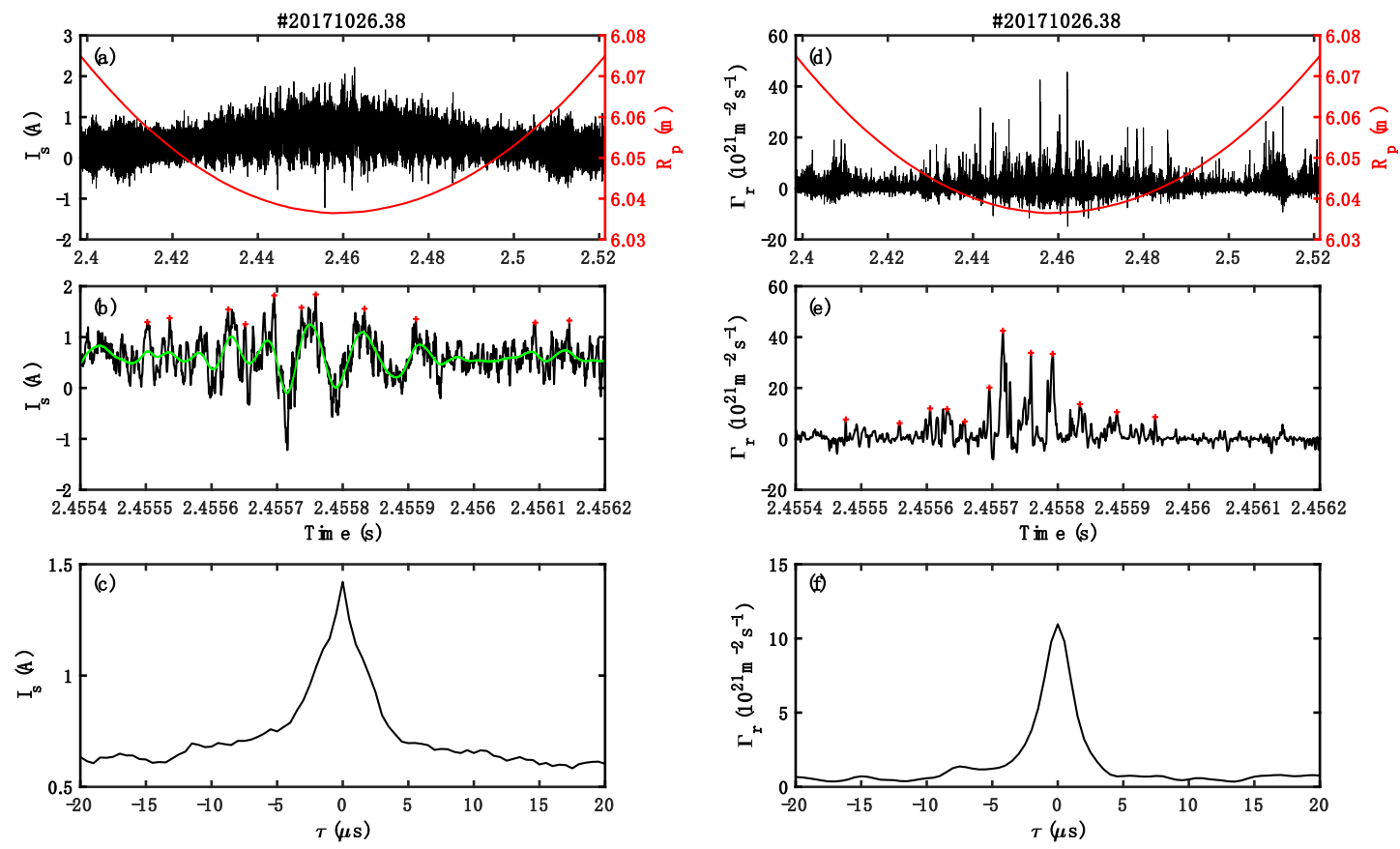

Figure 12. (a-b) Raw signal of the ion saturation current. The red crosses in (b) and (e) means that these spikes are selected by the conditional averaging procedure. The green line in (b) signifies the signal filtered by a $20 \mathrm{kHz}$ low-pass filter. (c) The conditional-averaged ion saturation current in the time period of $t=2.45-2.47 \mathrm{~s}$, i.e., $R=6.036-6.038 \mathrm{~m}$. (d-e) Raw signal of radial turbulent particle flux. (f) The conditional-averaged radial turbulent particle flux in the same time period as (c).
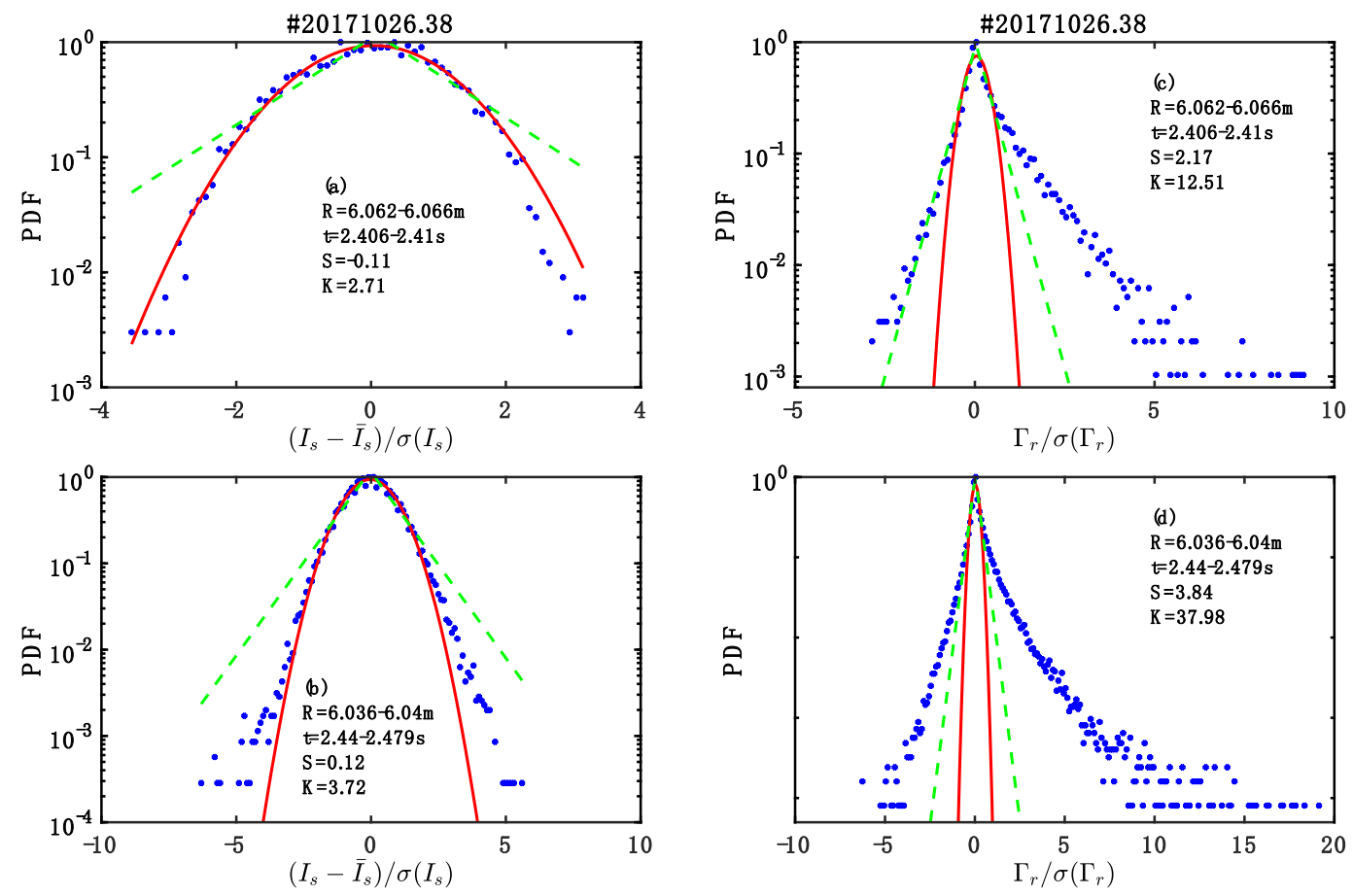

Figure 13. The probability distribution function (PDF) for the ion saturation current and the radial turbulent particle flux at two different radial positions. The skewness and kurtosis of the distribution are labelled in each panel. The red lines denote the Gaussian fitting for the PDF, and the green dashed lines denote the Laplace fitting. 


\subsection{Intermittent transport in the near SOL}

The radial transport is sharply reduced when $R<6.06 \mathrm{~m}$ and the dominant frequency changes from the $40-120 \mathrm{kHz}$ broadband spectrum to the $10-20 \mathrm{kHz}$ intermittent structures. Note that the word 'intermittent' here is used to signify the bursty events in the radial turbulence transport. As seen in Figure 7 and Figure 8, the radial evolution of radial transport and the $2 \mathrm{D}$ field line connection length are consistent with each other. In the short connection length region $R=$ $6.053-6.059 \mathrm{~m}$, it is a quiet region without intermittent events; during the radial region of $R=6.047-6.052 \mathrm{~m}$, the connection length becomes longer, at the same time the intermittent events start to appear but with lower amplitudes than the inner region; when the probe enters the radial region of $R=6.036-6.046 \mathrm{~m}$, the connection length continues to increase more and more quickly, and the intermittent transport gets more and more stronger and also the base line of the heat and particle fluxes. When $R<6.036 \mathrm{~m}$, the connection length increases rapidly and over $800 \mathrm{~m}$, and only the two floating potential pins $\phi_{f 3}$ and $\phi_{f 4}$ have measurements due to their inner positions. Figure 9(b) shows the APSD of the ion saturation current at the innermost point of the probe. The peak of the broadband spectrum disappears, instead, a low frequency turbulence centered at $15 \mathrm{kHz}$ has the highest power density. In the CPSD of two radially separated floating pins at the innermost point, the flat region of the broadband spectrum also disappears and exhibits a power decay factor of $\alpha=$ -2.34 . The maximum of the CPSD is clear at $f=15 \mathrm{kHz}$, indicating the radial propagation of the low frequency fluctuations. In Figure $10(\mathrm{~b}-\mathrm{d})$, there are three entirely different distribution of $S\left(k_{\theta}, f\right)$. In the radial region of $R=$ $6.043-6.053 \mathrm{~m}, S\left(k_{\theta}, f\right)$ is concentrated at $k_{\theta} \approx 0 \mathrm{~cm}^{-1}$ and symmetric about 0 . Note that the power spectral density in the low frequency is enhanced. In the radial region of $R=$ $6.034-6.041 \mathrm{~m}$, the power density below $20 \mathrm{kHz}$ gets much larger, and the broadband spectrum in $f=30-100 \mathrm{kHz}$ is directed to the electron diamagnetic drift direction with a group velocity about $V_{\text {group }} \approx 5 \mathrm{~km} / \mathrm{s}$ in the laboratory frame. As seen from Figure $7(\mathrm{~h})$, the radial electric field is about 1-6 kV/m when $R=6.034-6.041 \mathrm{~m}$, and the induced drift velocity $V_{E \times B}=0.6-3.8 \mathrm{~m} / \mathrm{s}$ directed in the ion diamagnetic drift direction. Consequently, the propagation velocity of this broadband spectrum is roughly $5.6-8.8 \mathrm{~km} / \mathrm{s}$ along the electron diamagnetic drift direction. However, the contribution to the radial particle flux from this broadband spectrum is much smaller than that from the low frequency turbulence, as shown in Figure 7(e). In the innermost point, $R=6.032-6.033 \mathrm{~m}$, the broadband spectrum above $30 \mathrm{kHz}$ becomes very weak and can be ignored, while most of the power density is concentrated in the low frequency turbulence that is centered at $15 \mathrm{kHz}$. The radial evolution of $S\left(k_{\theta}, f\right)$ is consistent with the variations of the radial turbulent transport, and has a strong dependence on the local magnetic topology

The properties of the intermittent transport are analyzed with a conditional averaging procedure which has been widely used in the analysis of intermittent events [50-52]. Totally 40000 points in the time slice $t=2.45-2.47 \mathrm{~s}$ are used for the conditional averaging, and the selected window of each fast event is set to $40 \mu \mathrm{s}$. A threshold of 2 times of the standard deviation of signal is used for this analysis. As shown in Figure 12(a), the raw signal of ion saturation current reveals strong intermittent events in the time period of $t=2.44-$ $2.48 \mathrm{~s}$, i.e., $R<6.04 \mathrm{~m}$. Note that the raw signal of $I_{s}$ exhibits some negative spikes though the mean value of $I_{s}$ is always positive. These negative $I_{s}$ spikes are also reported in MAST tokamak and linear plasma devices PISCES [53, 54]. In our experiment, a biasing voltage of $286 \mathrm{~V}$ is applied between both pins of the double probe, which is high enough to expel most of the electrons from the collected current. On the one hand, the transport of ions and electrons in the SOL of the island divertor configuration on the W7-X stellarator may lead to the negative $I_{s}$ fluctuations, as illustrated in the far SOL. On the other hand, the existence of fast electrons in the SOL due to the ECRH heating may also contribute to the negative spikes on $I_{s}$. Figure 12(b) gives an example of the data selection procedure, with the red cross denoting that the fast event is chosen in the ensemble for averaging. The green line shows the components of the signal below $20 \mathrm{kHz}$. A clear oscillation can be found on the green line, around a frequency of 10-20 kHz. During one circle of this oscillation, there are some spikes, such as the red crosses on the green hills. Figure 12 (c) is the conditional averaging $I_{s}$, having an amplitude about $1.4 \mathrm{~A}$ and the full width at half maximum (FWHM) is about $13 \mu \mathrm{s}$. The radial turbulent particle flux is also processed with the same conditional averaging method, as shown in Figure 12(d), (e) and (f). The maximum value of the radial particle flux is as high as $40 \times 10^{21} \mathrm{~m}^{-2} \mathrm{~s}^{-1}$ during the eruption of intermittent event. The conditional averaging $\Gamma_{r}$ peaks at $11 \times 10^{21} \mathrm{~m}^{-2} \mathrm{~s}^{-1}$ and has a FWHM of $3 \mu \mathrm{s}$.

The probability distribution functions (PDF) of the ion saturation current and radial turbulent particle flux are illustrated in Figure 13 [55]. Note that $\sigma\left(I_{s}\right)$ and $\sigma\left(\Gamma_{r}\right)$ are the standard deviation of $I_{s}$ and $\Gamma_{r}$, respectively. In the broadband dominant region ( $R=6.062-6.066 \mathrm{~m}$ ), the PDFs of $\left(I_{s}-\bar{I}_{s}\right) / \sigma\left(I_{s}\right)$ almost agree with the Gaussian fitting except that the points on the edge of two sides are located slightly below the fitting curve, with skewness of -0.11 and kurtosis of 2.71. The Gaussian distribution $f(x)=$ 
$a e^{-b x^{2}}$ has a skewness of 0 and kurtosis of 3 , which is similar to the PDFs of $\left(I_{s}-\bar{I}_{s}\right) / \sigma\left(I_{s}\right)$ in Figure 13(a); while the Laplace distribution $f(x)=a e^{-b|x|}$ has a skewness of 0 and kurtosis of 6 . In the intermittent transport region ( $R=$ $6.036-6.04 \mathrm{~m}$ ), PDFs of $I_{s}$ reveal elevated tails on both sides which are located between the curves of Gaussian fitting and the Laplace fitting, with skewness of 0.12 and kurtosis of 3.72. Based on the modelling of Hasegawa-Wakatatti turbulence, the PDFs of small events are well approximated by the Gaussian distribution, while the large events often exhibit a Laplace distribution [56]. The PDFs of $\left(I_{s}-\bar{I}_{s}\right) /$ $\sigma\left(I_{s}\right)$ in the broadband region are due to small scale turbulence, which is consistent with the observation in Figure 9(a) and Figure 13(a); while the PDFs in the near SOL are contributed by both small scale and large scale events, in consequence the elevated tails appear in Figure 13(b). In tokamaks the PDFs of $\left(I_{s}-\bar{I}_{s}\right) / \sigma\left(I_{s}\right)$ generally have positive skewness and heavy tail in the far SOL because of positive bursts, a near Gaussian distribution close to the LCFS, and a negative tail due to negative burst inside the velocity shear layer, such as JET [57], HL-2A [58] and J-TEXT [59]. In the stellarator W7-AS, Gaussian-like distribution of $\left(I_{s}-\bar{I}_{s}\right) / \sigma\left(I_{s}\right)$ is also observed near the LCFS [60]. In the SOL of W7-X with island divertor configuration, the transport is more complicated due to the three-dimension magnetic field structure, which may give rise to the near Gaussian distribution in the far SOL and the distribution with elevated tails in the near SOL. However, the symmetry of PDF in Figure 13(a) and (b) could be also related to the negative spike of $I_{s}$, which is needed to investigate further in the following experiments. The PDFs of $\Gamma_{r} / \sigma\left(\Gamma_{r}\right)$ display a strong asymmetry, with a large positive skewness $S=2.17$ for $R=6.062-6.066 \mathrm{~m}$ and $S=3.84$ for $R=6.036-$ $6.04 \mathrm{~m}$, indicating the intermittent transport is mainly positive. In the outer region, the PDFs on the left side agree very well with the Laplace fitting, but on the right side the points are located significantly above the Laplace fitting. In the inner region, the PDFs on both the left and right sides have much higher tails than that in the inner region, with a kurtosis $\sim 38$. The PDFs indicate the extremely large amplitude of an outward radial turbulent particle flux.

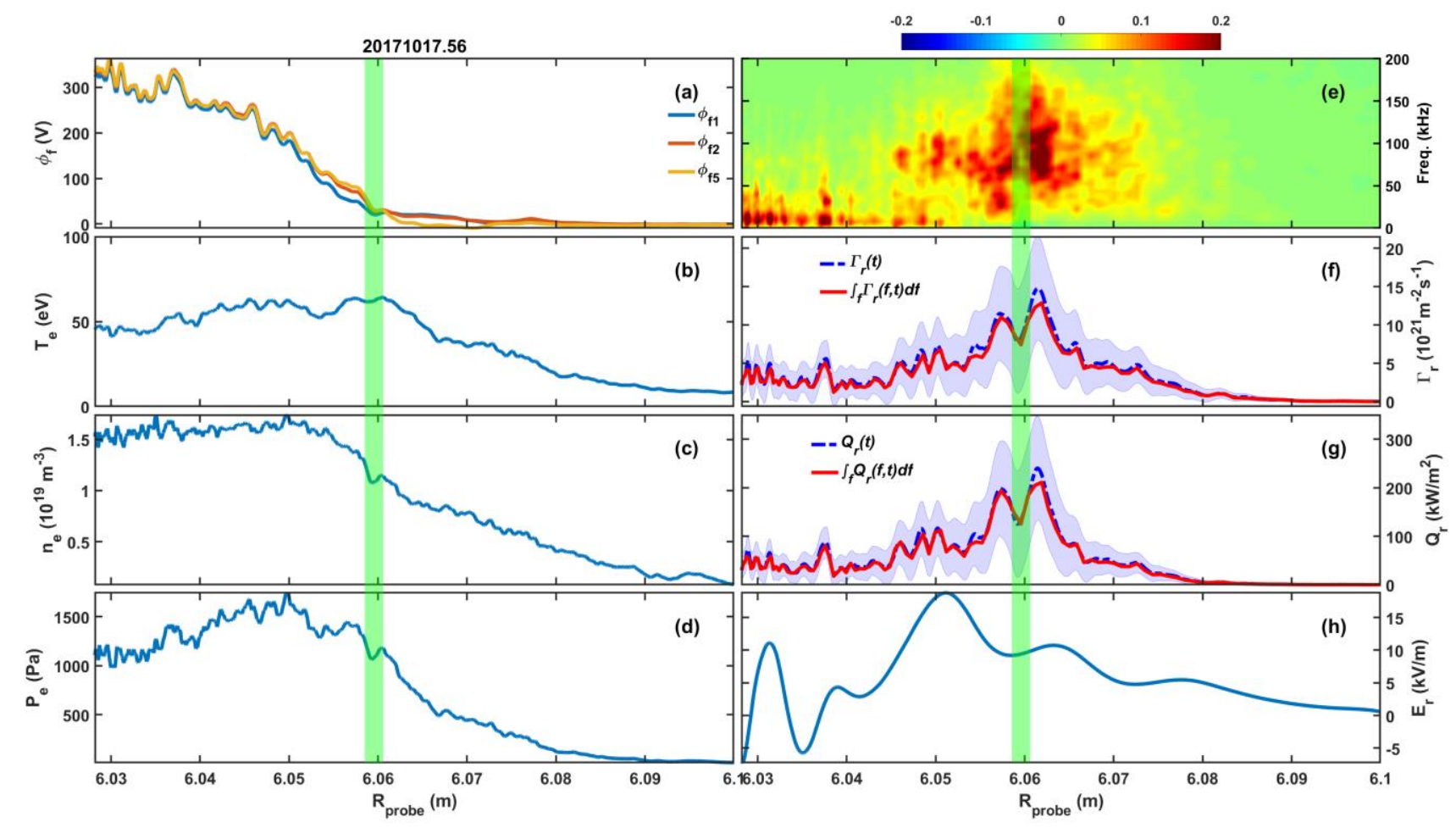

Figure 14. The radial plasma profiles, radial turbulent heat flux and particle flux of the high mirror configuration. The description is the same as Figure 7. 


\section{Turbulent transport in high mirror configurations}

In the high mirror configuration $(\mathrm{KJM}+252)$, the distance between the innermost point of probe and the LCFS is $d R=$ $6.022 \mathrm{~m}-6 \mathrm{~m}=22 \mathrm{~mm}$, which is much larger than that in the standard configuration. The heat and particle fluxes in high mirror configuration are shown in Figure 14. Since the width of the SOL island is distinctly wide in the $\mathrm{KJM}+252$ configuration, the broadband spectrum in Figure 4(f) covers a wide radial region, and also contributes to the outward radial turbulent particle flux, as presented in Figure 14(e). In the region of $R=6.047-6.073 \mathrm{~m}$, the radial particle flux is dominated by a broadband turbulence from $40 \mathrm{kHz}$ to 160 $\mathrm{kHz}$, and highlighted in the region of $R=6.06-6.063 \mathrm{~m}$. When $R<6.051 \mathrm{~m}$, the intermittent transport starts to appear and gets much clearer when probe plunging deeper. The smooth radial turbulent particle flux $\Gamma_{r}$ calculated from equation (1) peaks at $R=6.061 \mathrm{~m}$ and $R=6.057 \mathrm{~m}$ with the maximum value about $15 \times 10^{21} \mathrm{~m}^{-2} \mathrm{~s}^{-1}$, which is in good agreement with the $\int \Gamma_{r}(f, t) d f$ derived from equation (3). The radial turbulent heat flux calculated from equation (2) is also consistent with that from equation (4), peaking at the same radial positions with a maximum value of $0.24 \mathrm{MWm}^{-2}$. It should be pointed out that the radial particle and heat fluxes without smoothing can be up to $90 \times 10^{21} \mathrm{~m}^{-2} \mathrm{~s}^{-1}$ and 1.7 $\mathrm{MW} \mathrm{m}^{-2}$ during the eruption of fast events, respectively. In the broadband dominant region, the electron density, temperature and pressure have a steep gradient, as shown in Figure 14 (bd). For the highlighted region $(R=6.06-6.063 \mathrm{~m}), n_{e}, T_{e}$ and $P_{e}$ have a steep gradient. While for the second highest peak region $(R=6.056-6.059 \mathrm{~m})$, only the $n_{e}$ and $P_{e}$ have a large gradient. However, in this highlighted region, the radial electric field is almost constant about $10 \mathrm{kV} / \mathrm{m}$. Compared to the 2D field line connection length in Figure 15, the connection length $L_{\|}$jumps from $25 \mathrm{~m}$ to $250 \mathrm{~m}$ at $R=$ $6.063 \mathrm{~m}$, simultaneously the radial heat and particle fluxes reach their maximums. When the connection length has a drop at $R=6.053 \mathrm{~m}$, the intermittent events start to appear, and the profiles of $n_{e}, T_{e}$ and $P_{e}$ enter a region with slow variation. Near $R=6.04 \mathrm{~m}$ there is a protuberance on the connection length, then $L_{\|}$is raised to a level with longer length, meanwhile the intermittent turbulence is predominant and the broadband spectrum disappears. The integrals of particle flux in the frequency range of $5-30 \mathrm{kHz}$ and $40-160$ $\mathrm{kHz}$ are calculated and shown in Figure 16(c). The ratio of $\int_{40 \mathrm{k}}^{160 \mathrm{k}} \Gamma_{r}(f, t) d f / \int_{1}^{400 \mathrm{k}} \Gamma_{r}(f, t) d f$ from the broadband turbulence possesses $60-80 \%$ of the total radial turbulent particle transport in the radial region of $R=6.05-6.08 \mathrm{~m}$. While the ratio of $\int_{5}^{30 \mathrm{k}} \Gamma_{r}(f, t) d f / \int_{1}^{400 \mathrm{k}} \Gamma_{r}(f, t) d f$ from the low frequency turbulence occupies more than $40 \%$ of the total radial turbulent particle transport in the radial region of $R<6.04 \mathrm{~m}$. Similar to the relative fluctuation amplitudes of electron density and temperature in the standard configuration, the ratio of $\left(\delta n_{e} / n_{e}\right) /\left(\delta T_{e} / T_{e}\right)$ is around 1014 in the broadband dominant region, as shown in Figure 16(b). In Figure 9, the APSD of the ion saturation current in the $\mathrm{KJM}+252$ configuration reveals similar features as the EJM+252 configuration, except that the broadband spectrum has a wider frequency distribution at $R=6.06 \mathrm{~m}$ and the peak of the APSD is shifted to a smaller frequency slightly at $R=6.026 \mathrm{~m}$. These two features are also clear in the CPSD of $\phi_{f 2}$ and $\phi_{f 3}$. The relative fluctuation amplitudes of the fast events in the intermittent transport region of the high mirror configuration are not as large as that in the standard configuration, because the probe is still far away from the LCFS in the high mirror configuration. However, the statistics of the intermittent events exhibits similar characteristics in PDFs, skewness and kurtosis.

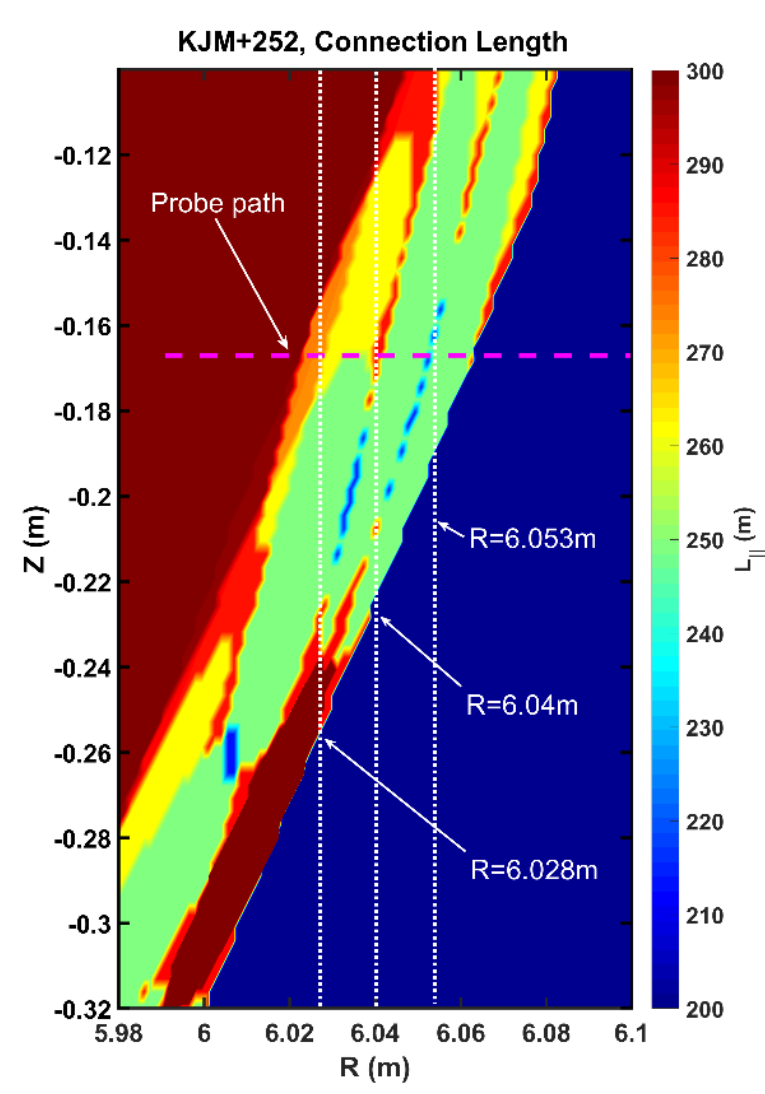

Figure 15. Two-dimension field line connection length for the $\mathrm{KJM}+252$ configuration in the poloidal plane of probe. 


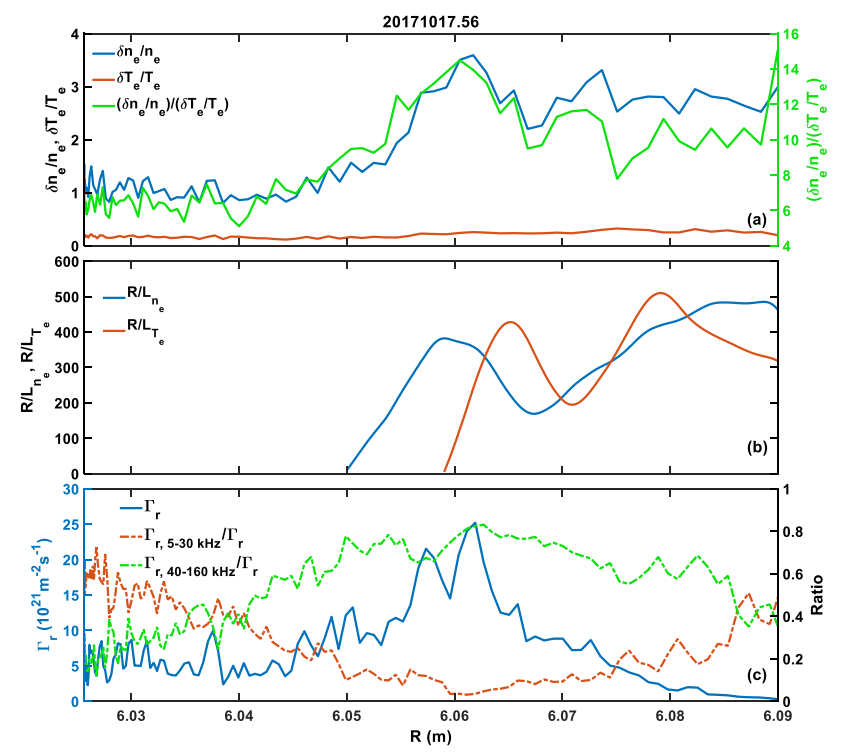

Figure 16. (a) Relative fluctuation levels of electron density and temperature. (b) Normalized gradient scale length of density and temperature. (c) Radial turbulent particle flux, the ratio of particle flux contributed by $5-30 \mathrm{kHz}$ turbulence to the particle flux of the whole frequency, and the ratio of $\Gamma_{r}$ from $40-160 \mathrm{kHz}$ to the total particle flux.

\section{Discussion and conclusion}

A new combined probe designed to measure the edge plasma profiles and turbulence structures has been developed and used in OP1.2a on W7-X. The plasma profiles, turbulence structure and radial transport are measured by this probe head for the standard and high mirror configuration. The plasma profiles and the radial variations of the auto-correlation power spectrum are in good agreement with the magnetic topology, including the SOL magnetic island structure and the connection length calculated by a field line tracer. Both the standard and high mirror configurations reveal two distinct turbulence transport patterns in the SOL: the first one located in the outer region is contributed by a broadband turbulence; the second one in the near SOL is induced by low frequency turbulence. In the standard configuration the probe has the deepest plunge with a distance of $6 \mathrm{~mm}$ to the LCFS, therefore the transport behavior in the EJM+252 configuration is analyzed in detail.

In the standard configuration, the radial turbulent heat flux and particle flux are calculated by two methods, i.e., one from the time domain analysis and the other from the frequency domain analysis. The results from both methods are in quite good agreement with each other. The radial turbulent particle flux is driven by a broadband turbulence with a frequency range between $40-120 \mathrm{kHz}$ in the radial region of $R=6.06-$ $6.065 \mathrm{~m}$ and $R=6.07-6.073 \mathrm{~m}$. Note that between these two radial regions the broadband turbulence is mitigated and also the radial transport. In the APSD of ion saturation current, the broadband spectrum highly peaks at the frequency about $70 \mathrm{kHz}$. The relative fluctuation amplitude of the electron density is about 1.2 in the broadband dominant region, but the relative fluctuation amplitude of the electron temperature is only 0.1 , giving a ratio $\left(\delta n_{e} / n_{e}\right) /\left(\delta T_{e} / T_{e}\right) \approx 10$. In this region the electron density normalized gradient scale length $R / L_{n_{e}}$ is also large. The particle effective diffusion coefficient estimated for the broadband turbulence is about 1$2.3 \mathrm{~m}^{2} \mathrm{~s}^{-2}$. From the statistical poloidal correlation power spectrum $S\left(k_{\theta}, f\right)$, the propagation velocity of the broadband turbulence is about $4.6 \mathrm{~km} / \mathrm{s}$ along the ion diamagnetic drift direction in the laboratory frame, and about $2.3-4.4 \mathrm{~km} / \mathrm{s}$ in the same direction in the plasma frame. The important turbulence parameter $k_{\theta} \rho_{s} \approx 0.07-0.08$, which is close to 0.1 . The radial heat and particle fluxes in the high mirror configuration are much larger than those in the EJM+252 configuration due to higher heating power, and also exhibit strong dependence on the local magnetic topology. However, the primary features of radial turbulent transport in the $\mathrm{KJM}+252$ configuration are consistent with those in the EJM+252 configuration. The principal characters of the broadband dominant transport can be listed as follows. (1) The smooth radial heat and particle fluxes driven by turbulence are directed outwards. (2) The frequency range of the broadband spectrum is from several tens of $\mathrm{kHz}$ to more than $100 \mathrm{kHz}$. (3) The broadband turbulence propagates along the ion diamagnetic drift direction with a velocity about $2.3-4.4 \mathrm{~km} / \mathrm{s}$ in the plasma frame. (4) The poloidal wavenumber near the central frequency of the broadband spectrum is $\sim 1 \mathrm{~cm}^{-1}$ and $k_{\theta} \rho_{s}$ is close to 0.1. (5) The high radial turbulent transport region is accompanied by a steep electron density gradient. (6) The relative fluctuation amplitude of the electron density is about 10 times larger than that for the electron temperature. In addition, the ion diamagnetic frequency in the laboratory frame is about $60 \mathrm{kHz}$ which is close to the central frequency of the broadband turbulence. As we know from the drift wave turbulence theory and experiment, terms (2-4) match the conditions of ITG drift wave instability, but usually ITG has much larger fluctuations in temperature than the fluctuation in density $[3,4,61]$. Furthermore, there is no ion information in our measurements. It would be better to leave the identification of the mode mechanism to future work. In consequence, we prefer to present the experimental results in this manuscript and shed more light on the investigation of the SOL turbulence in the new optimized stellarator Wendelstein 7-X. In future experiments, the new combined probe head will be collaborated with other diagnostics (e.g., retarding field analyzer (RFA)) to measure the information of both ions and electrons.

The intermittent transport behavior is analyzed with some statistical techniques. In both the standard and high mirror 
configurations, the intermittent transport is located at the radial position between the broadband spectrum and the LCFS. The conditional averaging method is used to illustrate the properties of fast events on the ion saturation current and radial turbulent particle flux. The conditional averaging of $I_{S}$ has a FWHM of about $13 \mu \mathrm{s}$, which is similar to the time scale of blobs in some fusion devices [62, 63]. Besides these fast events, an obvious oscillation around $10-20 \mathrm{kHz}$ is accompanied with the fast events, and the turbulence within this frequency range dominates the radial transport as shown in the frequency distribution of $\Gamma_{r}(f, R)$. The PDFs of the fluctuations of the ion saturation current obey the Gaussian distribution in the broadband turbulence dominant region, while elevated tails between the Gaussian distribution and the Laplace distribution in the intermittent transport region are revealed. Note that the negative spikes in the signal of ion saturation current may lead to this Gaussian-like distribution, which is needed to further investigate in the following experiments on W7-X. In both radial regions the PDFs of $\Gamma_{r}$ are mainly on the positive side, with skewness larger than 2 .

To sum up, the SOL turbulence structure and transport exhibit a strong dependence on the local magnetic topology in W7-X. It is important to investigate the topology effects on radial heat and particle transport, which will directly influence the heat load on the divertor plates. This article shows the turbulence induced transport exhibiting two remarkable dominant mechanisms and their close relationship with connection length of field lines, and is beneficial to the edge turbulence theory and simulation. In addition, the broadband turbulence dominant radial transport in the far SOL switches to the low frequency dominant transport in the near SOL, which is caused not only by the local magnetic topology but also the parallel transport through the stochastic open field lines. An edge transport model for the island divertor configuration of W7-X should be developed to illustrate the radial transport and parallel transport in the 3D field line system. Furthermore, comparisons of the edge plasma characteristics and transport between $\mathrm{W} 7-\mathrm{X}$ and tokamaks are important to understand the impact of the magnetic perturbations on the edge plasma confinement and transport. This paper will shed more light on these aspects from the experiments.

\section{Acknowledgements}

The authors gratefully acknowledge the discussion and support from Michael Endler, Yumin Wang and Liang Wang. This work has been carried out within the framework of the EUROfusion Consortium, has received funding from the Euratom research and training programme 2014-2018 under grant agreement No 633053, and was supported by the National Natural Science Foundation of China under Grant Nos. 11875294, 11675211, 11605235 and 11405213. The views and opinions expressed herein do not necessarily reflect those of the European Commission.

\section{References}

[1] Horton W. 1999 Rev Mod Phys 71735

[2] Horton W. 1990 Phys Rep 1921

[3] Tynan G. R. et al 2009 Plasma Phys. Control. Fusion. 51 113001

[4] Doyle E. J. et al 2007 Nucl. Fusion 47 S18

[5] Zweben S. J. et al 2002 Phys. Plasmas 91981

[6] Maqueda R. J. et al 2001 Rev. Sci. Instrum. 72931

[7] Shesterikov I. et al 2013 Rev. Sci. Instrum. 84053501

[8] Liu S. C. et al 2012 Rev. Sci. Instrum. 83123506

[9] Xu G. S. et al 2014 Nucl. Fusion 54103002

[10] Boedo J. A. et al 2003 Phys. Plasmas 101670

[11] Lampert M. et al 2018 Phys. Plasmas 25042507

[12] Grulke O. et al 2001 Phys. Plasmas 85171

[13] Silva C. et al 2009 J. Nucl. Mater. 390-91 355

[14] Nie L. et al 2014 Plasma Phys. Control. Fusion. 56 055006

[15] Muller H. W. et al 2011 Nucl. Fusion 51073023

[16] Tanaka H. et al 2015 J. Nucl. Mater. 463761

[17] Fuchert G. et al 2016 Plasma Phys. Control. Fusion. 58 054005

[18] Fuchert G. et al 2013 Plasma Phys. Control. Fusion. 55 082309

[19] Garcia O. E. et al 2006 Phys. Plasmas 13082309

[20] Ida K. et al 2015 Nat Commun 65816

[21] Ida K. et al 2015 Plasma Phys. Control. Fusion. 57

014036

[22] Castejon F. et al 2016 Plasma Phys. Control. Fusion. 58 094001

[23] Zoletnik S. et al 2002 Plasma Phys. Control. Fusion. 44 1581

[24] Liu S. C. et al 2018 Phys. Plasmas 25072502

[25] Liu S. C. et al 2018 Nucl. Fusion 58046002

[26] Pedersen T. S. et al 2016 Nat Commun 713493

[27] Satheeswaran G. et al 2017 Fusion Eng. Des. 123699

[28] Nicolai D. et al 2017 Fusion Eng. Des. 123960

[29] Brunner D. et al 2013 Rev. Sci. Instrum. 84053507

[30] Ezumi N. et al 2005 J. Nucl. Mater. 3371106

[31] Ezumi N. et al 2003 J. Nucl. Mater. 313696

[32] Rummel T. et al 2018 Ieee T Plasma Sci 461517

[33] Geiger J. et al 2015 Plasma Phys. Control. Fusion. 57 014004

[34] Bozhenkov S. A. et al 2013 Fusion Eng. Des. 882997

[35] Grahl M. et al 2017 Fusion Eng. Des. 1231015

[36] Grahl M. et al 2018 Ieee T Plasma Sci 461114

[37] Hutchinson I. H. 2002 Principles of plasma diagnostics (Cambridge ; New York: Cambridge University Press)

[38] Liang Y. et al 2007 Phys. Rev. Lett. 98265004

[39] Zhao K. J. et al 2015 Nucl. Fusion 55073022

[40] Xu Y. et al 2007 Nucl. Fusion 471696

[41] Schirmer J. et al 2006 Nucl. Fusion 46 S780

[42] Burrell K. H. 1997 Phys. Plasmas 41499

[43] Xu X. Q. et al 2002 Nucl. Fusion 4221

[44] Beall J. M. et al 1982 J. Appl. Phys. 533933 
[45] Levinson S. J. et al 1984 Nucl. Fusion 24527

[46] Cordey J. G. et al 1996 Plasma Phys. Control. Fusion.

381905

[47] Berk H. L. et al 1993 Nucl. Fusion 33263

[48] Wang H. Q. et al 2014 Phys. Rev. Lett. 112185004

[49] Ritz C. P. et al 1989 Phys. Rev. Lett. 621844

[50] Grulke O. et al 1999 Phys. Plasmas 6788

[51] Boedo J. A. et al 2001 Phys. Plasmas 84826

[52] Xu G. S. et al 2009 Nucl. Fusion 49092002

[53] Antar G. Y. 2004 Contrib. Plasm. Phys. 44217

[54] Antar G. Y. et al 2003 Phys. Plasmas 10419

[55] D'Ippolito D. A. et al 2011 Phys. Plasmas 18060501

[56] Anderson J. et al 2017 Phys. Plasmas 24062301

[57] Xu G. S. et al 2010 Phys. Plasmas 17022501

[58] Cheng J. et al 2010 Plasma Phys. Control. Fusion. 52 055003

[59] Zhu M. Z. et al 2011 Chinese Phys B 20025204

[60] Simon P. et al 2014 Plasma Phys. Control. Fusion. 56 095015

[61] Horton W. et al 2008 Turbulent Transport in Fusion Plasma 10131

[62] Kocan M. et al 2013 Nucl. Fusion 53073047

[63] Xu Y. H. et al 2005 Plasma Phys. Control. Fusion. 47 1841 